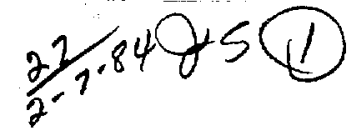

\title{
DR 2096-6
}

LBI, -16578

\section{Lawrence Berkeley Laboratory UNIVERSITY OF CALIFORNIA}

\section{EARTH SCIENCES DIVISION}

TECHNIQUES FCR MEASURING THE VERTICAL HYDRAULIC CONDUCTIVITY OF FLOOD BASALTS AT THE BASALT

WASTE ISOLATION PROJECT SITE

I. Javandel

LBL- -16578

DE84 006262

June 1983

MASIER

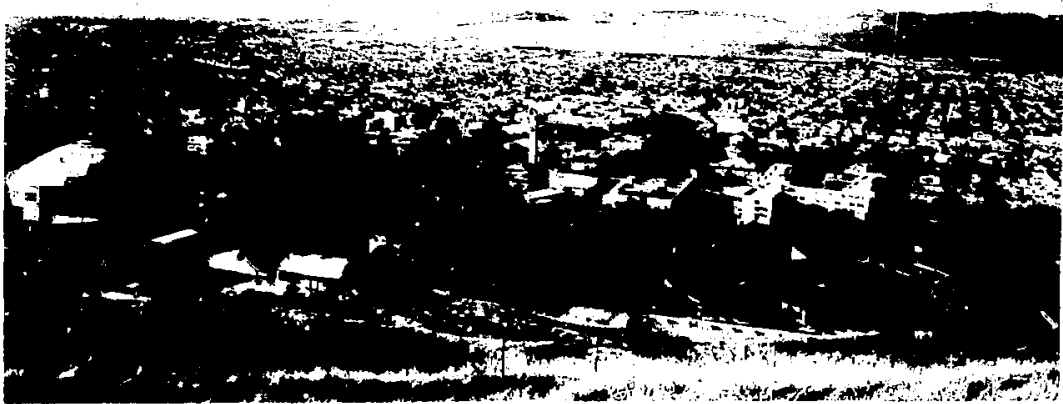

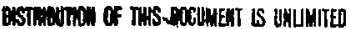




\title{
DISCLAIMER
}

This report was prepared as an account of work sponeored by an seensy of the Unived States Gowernment. Neither the United States Governorent nor any abacy thereof, oor any of their employes, mates any warranty, expreas or implied, or asumes any lepol biability or recpowibility for the ccouracy, esoptetenest, or usefulness of any informatios, apsarates prodect, or process discloed, or represents that its use woukd not isfringe privately owied rivtuts. Reference herein to any specific commercial product, process, or service by trade asioc, tradeinatl, manufacturer, or otherwise does not neocssarily constitute or imply its eadoresenent, reommendation, or favoring by the United States Gowemment or any atency theroof. The view and opinions of authors expreseed herein do not necesarily state or reflect those of the United States Govemment or any agency thereof.

TECHNIQUES FOR MEASURING THE VERTICAL HYDRAULIC CONDUCTIVITY
OF FLOOD BASALTS AT THE BASALT WASTE ISOLATION PROJECT SITE

\author{
Iraj Javardel \\ Lawrence Berkeley Iaboratory \\ University of California \\ Berkeley, California 94720
}

June, 1983

This work was supported by the Rockwell Hanford Operations Basalt Waste

Isolation Project under U.S. Department of EnergY Contract DE-AC03-76SF00098. 
NOMENCLATURE . . . . . . . . . . . . . . . . . . . . . iii

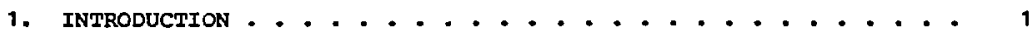

1.1 objective ....... . . . . . . . . . . . . . . . 1

1.2 Statenent of Froblem . . . . . . . . . . . . . . 1

1.3 Hydraulic Conductivity ................. 1

2. AVAILAELE testing techigues . . . . . . . . . . . . . . 5

2.1 Tests based on the Theory of Partial Penetration . . . . 5

2.1.1 Weeks' Method ................... 5

2.1.2 Shao-McKee Method .. ............. . 9

2.2 Tests Based on the Theory of Leaky Aquifers . . . . . . . . 13

2.2.1 Hantush and Jacob Solution... . . . . . . . 13

2.2.2 Hantush Modifiez Solution . . . . . . . . . . 20

2.2.3 Witherspoon and Neuman Ratio Method . . . . . . . 27

2.3 Tests Based on the Concept of Directional Hydraulic

Conductivity . . . . . . . . . . . . . . . . 36

2.3.1 Single Well Tests ............... . 45

3. COMParison of aVaILABLE techniques . . . . . . . . . . . . . 49

4. RECOMMENDED METHODS .. . . . . . . . . . . . . . . . . 52 
NOMENCLATURE
a $\quad=\frac{1}{|K|} \cdot\left(L^{-3} r^{3}\right)$
B $\quad=\sqrt{\frac{K b^{\prime}}{K^{\prime}}}$, leakaye factor, (L)
$b, b^{\prime}=$ Thickness of an aquifer and aquitard, respectively, (L)
$\operatorname{erfc}(x)=1-\frac{2}{\sqrt{\pi}} \int_{0}^{\infty} \bar{e}^{y^{2}} d y$, complementary error function
g = acceleration of gravity, (LT $\mathrm{T}^{-2}$ )
h $\quad=$ hydraulic head, (L)
$K^{\prime} \mathrm{K}^{\prime} \quad=\quad$ hydraulic conductivities of an aquifer and aquitard, respec- tively, $\left(\mathrm{LT}^{-1}\right)$
$|k|=$ determinant of hydraulic conductivity tensor, $\left(\mathrm{L}^{3} \mathrm{~T}^{-3}\right)$
$\operatorname{det}\left(\mathrm{K}_{\mathrm{i} j}\right)=$ determinant of tensor $\mathrm{k}_{\mathrm{i} j}$
$K_{\mathrm{d}}=$ directional hydraulic conductivity along the flow
$\mathrm{K}_{\mathrm{r}}, \mathrm{K}_{\mathbf{Z}} \quad=$ components of hydraulic conductivity in radial and vertical directions, respectively, (LT $\left.{ }^{-1}\right)$
$\mathbf{k}_{x x}, k_{y y}$
$=$ components of hydraulic conductivity tesnor (LT ${ }^{-1}$ )
$k_{0}$
$=$ modified Bessel function of the second kind and zero order
k = intrinsic permeability, $\left(L^{2}\right)$
1 depth of penetration, (I)
$M, M^{\prime} \quad=$ thickness of an aquifer and aquitar, respectively, (L)
$Q=$ rate of pumping or recharge, $\left(\mathrm{L}^{3} \mathrm{~T}^{-1}\right)$
$r \quad=\quad$ radial distance from a pumping well, (L)
$r_{c} \quad=\quad r \sqrt{\frac{K_{2}}{K_{r}}},\langle L\rangle$ 
$\mathbf{S}^{\prime} \mathbf{s}^{\prime} \quad=$ storage coefficient of an aquifer and aquitard, respectively

$S_{S}, S^{\prime} \mathbf{S}=$ specific storage of an aquifer and aquitard, respectively, (t-1)

s,s' $\quad$ drawdowns in an aquifer and aquitard, respectively, (L)

$s_{D} \quad x$ dimensionless drawdown

$T_{X x} \quad x \quad K_{x x^{b},\left(L^{2} T^{-1}\right)}$

$\mathrm{T}_{\mathrm{YY}} \quad=\quad \mathrm{K}_{Y Y^{\mathrm{b}}},\left(\mathrm{L}^{2} \mathrm{r}^{-1}\right)$

$\mathrm{T}_{\mathrm{xy}} \quad=\quad \mathrm{K}_{\mathrm{xy}} \mathrm{b},\left(\mathrm{L}^{2} \mathrm{~T}^{-1}\right)$

$t \quad=$ time, (T)

$t_{D} \quad=$ dimensionless time

$t_{D}^{\prime} \quad=\frac{K^{\prime} t}{s_{s}^{\prime} z^{2}}$

$u=\frac{r^{2} s s}{4 K t}$

$\mathrm{v} \quad=\quad$ specifie discharge or Darcy's velocity, $\left(\mathrm{LT}^{-1}\right.$ )

$v_{\mathbf{X}^{\prime}} v_{y^{\prime}}=$ components of specific discharge, $\left(\mathrm{LT}^{-1}\right)$

$w(u) \quad=\int_{u}^{\infty} \frac{e^{-y}}{y} d y$, well function

$w(u, a)=\int_{u}^{\infty}\left(\frac{1}{y}\right) \exp \left(-y-\frac{a^{2}}{4 y}\right) d y$

$B_{1}, B_{2}, B_{3}=$ the angles between the direction of specific discharge and $x, y, z$ axes, respectively

o $\quad$ density of fluid $\left(\mathrm{ML}^{-3}\right)$

$\mu \quad=$ dynamic viscosity, $\left(\mu^{-2} \mathrm{~T}^{-1}\right)$ 


\section{INTRODUCTION}

\subsection{Objective}

This report compares available techniques for measuring vertical hydraulic conductivity in geological materials and identifies methods that are most applicable to the needs of the Basalt Waste Isolation Project (BwIP). We define vertical hydrauiic conductivity as the hyaraulic conductivity in the direction normal to the bedding plane.

\subsection{Statement of the Problem}

A regional model that can predict groundwater movement through the reference repository location and surrounding area is essential to assessing the site switability for a nuclear waste repository. vuring the last two decades, several models have been developed to handle complicated flow patterns through complex geologic materiais. The basic problem, however, is obtaining the data base needed to apply these models.

The hydological data needed include the spatial distribution of effective porosity, the hydraulic conductivity tensor and its variation in space, values of specific storage, the hydraulic head distribution, and the fluid properties. In this report, we discuss conventional methods of obtaining vertical hydraulic conductivity and examine their applicability to the BWIP site.

\subsection{Hydraulic Conductivity}

Hydraulic conductivity is the constant of proportionality in Darcy's Law that relates groundwater movement and hydraulic gradicin in a porous medium. Darcy's Law may be stated as:

$$
V=-K \frac{d h}{d x}
$$


where

$$
\begin{aligned}
& K=\text { hydraulic conductivity, } \\
& v=\text { Darcy's velocity or specific discharge, and } \\
& \frac{d h}{d x}=\text { hydraulic gradient }
\end{aligned}
$$

Hydraulic conductivity, which is sometimes called the coefficient of permeability, has been shown to be relatod to the fluid properties and the permeability of a porous medium by the following formula (Hubbert, 1940):

$$
\mathrm{K}=\frac{\mathrm{k} \rho \mathrm{g}}{\mu}
$$

where

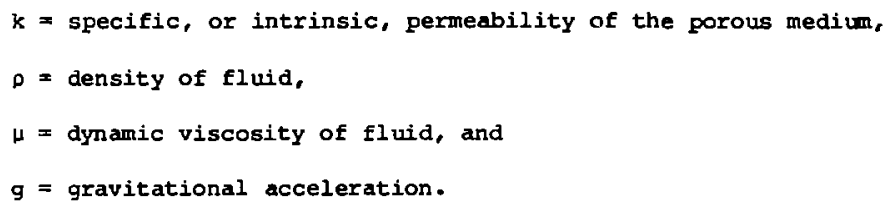

Intrinsic permeability $k_{i}$ wijich is a function of mean grain diameter, grain size distribution, sphericity, and roundness of the grain; is a measure of the ability of the medium to transfer a given fluid.

The hydraulic conductivity of geological materials varies from approximately 1 to $10^{-13} \mathrm{~m} / \mathrm{s}$. The hydraulic conductivity of a given geological formation can vary with the location of measurement. This property of a medium is called heterogenity. Hydraulic conductivity can also vary with the direction of measurement at any given locaton. This property is called anisotropy and is quite conmon in sedimintary rocks. In such rocks hydraulic conductivity parallel to the bedding is soinetimes much larger than across the bedding. fnisotropy is especially inportant in stratified formations. 
Rose (1983) discusses how directional hydraulic conductivity is affected by thickness and differences between adjacent layers in a sedimentary rock. Low hydraulic conductivity beds within a more permeable medium do not caluse significant anisotropy unless the total thickness of the low conductivity beds is of the same order as the thickness of the permeable materials. The maximum value of the ratio $K_{\max } / K_{\min }$ corresponds to the case where the total thickness of each material is equal. Anisotropy is also common in fractured rocks where the aperture and spacing of joints varies with direction.

In an anisotropic medium, hydraulic conductivity may generally be represented by a $3 \times 3$ matrix. The components of specific discharge in an anisotropic medium may then be written.

$$
\begin{aligned}
& v_{x}=-k_{x x} \frac{\partial h}{\partial x}-k_{x y} \frac{\partial h}{\partial y}-k_{x z} \frac{\partial h}{\partial z} \\
& v_{y}=-k_{y x} \frac{\partial h}{\partial x}-k_{y y} \frac{\partial h}{\partial y}-k_{y z} \frac{\partial h}{\partial z} \\
& v_{z}=-k_{z x} \frac{\partial h}{\partial x}-k_{z y} \frac{\partial h}{\partial y}-k_{z z} \frac{\partial h}{\partial z}
\end{aligned}
$$

Values of $\mathrm{X}$ are components of the symmetric hydraulic conductivity matrix. With an appropriate coordinate system one can diagonalize a symmetric matrix if the principal directions of hydraulic conductivity coincide with the $x, y$, and $z$ coordinate axes. If the matrix can be simplified, then the three principal components of specific discharge are represented by:

$$
\begin{aligned}
& v_{x}=-k_{x} \frac{\partial h}{\partial x} \\
& v_{y}=-k_{y} \frac{\partial h}{\partial y}
\end{aligned}
$$




$$
v_{z}=-K_{z} \frac{\partial h}{\partial z}
$$

Here $k_{x}, k_{y}$, and $k_{z}$ are the principal hydraulic conductivity values which are now in the $x, y$, and $z$ directions. Equations (1-6) through (1-8) indicate that for an anfsotropic medium, the orientations of specific discharge and hydraulic gradient coincide only in the princlpal directions of hydraulic conductivity. In other directions, however, the hydraulic gradient and specific discharge are not in the same orientation.

It is often possible to choose a coordinate systen that coincides with the princtpal directions of hydraulfc conductivity. But in layered fractured media, where fracture orintation and spacing may change from one layer to another, the principal direction of conductivity may also change. In such a medium it is impossible to identify a unique system of coordinates. Therefore, study of flow in such a medium requires identification of the complete hydraulic conductivity tensor of the individual layers. As will be discussed in section 2, most methods for measuring vertical conductivity do not permit the complete conducttvity tensor to be identified because they require the a priori assumption that the vertical direction is a principal direction of hydraulic conductivity.

A general assumption used in all the testing techniques discussed in this report is that the formation being tested is a porous medium. These tests can be performed in a fractured rock provided that the fracture system is well developed such that it can be represented by an equivalent porous medium. 
2. AVALLABLE TESTING TEChNIqUES

There are three types of testing methods for pertical hydraulic conductivity: (1) tests associated with partially penetrating production wells, (2) tests related to the leaky aquifers, and (3) tests which lead to calculation of directional hydraulic conductivity.

\subsection{Tests Based on The Theory of Partial Penetration}

If a pump test is designed so that a horizontal aquifer is pumped by a vertical well, draking from the total thickness of the aquifer, the vertical hydraulic conductivity of the aquifer cannot be estimated because groundwater movement is essentially horizontal. However, analysis of drawdown data from a well that only partially penetrates an aquifer may yield the vertical hydraulic conductivity if drawdown data is obtained from a point close to the pumped well.

Weeks (1969) presented methods for determining the ratio of horizontal to vertical hydraulic conductivity. These methods are based on the assumption that the horizontal hydraulic conductivity in the aquifer is independent of direction. Shao and McKee (1982) introduced another method for determining all three principal values of hydraulic conductivity provided that they coincide with a coordinate system that has two axes on the bedding plane and the third perpendicular to that plane.

\subsubsection{Weeks: Method}

Heeks' method is based upon the partial penetration theory of Hantush (1957, 1961, 1964). The drawdown around a partially penetrating well producing at a constant rate from a a homogeneous, anisotropic aquifer is given by: 


$$
s=\frac{o}{4 \pi k_{r} b}\left\{w_{r} u_{I}+f\right\}
$$

where

$$
f=\frac{2 b}{a(I-d)} \sum_{n=1} \frac{1}{n}\left(\sin \frac{n \pi l}{b}-\sin \frac{n \pi d}{b}\right) \cos \frac{n \pi z}{b} w\left(x^{\prime}, \frac{n \pi}{b} \sqrt{k} z^{\prime}\right)
$$

and

$$
\begin{aligned}
& u_{r}=\frac{r^{2} s_{s}}{k k_{r} b t} \\
& \text { s } \quad \text { draitdown } \\
& S_{\mathrm{S}} \quad=\quad \text { megiflc storage } \\
& r \quad \text { = radial distance } \\
& \mathrm{K}_{\mathrm{Y}}, \mathrm{K}_{\mathrm{z}} \quad=\text { hydraulic conductivity in radial and vertical direction, } \\
& \text { respectively } \\
& \text { b = thichess of the aquifer } \\
& t \quad=\quad \text { time elapsed since the start of the punp tast } \\
& \omega\left(u_{r}, \frac{n \pi r}{b} \sqrt{k_{z} / k} r\right)=\omega(u, \alpha)
\end{aligned}
$$

are defined in the nomenclature.

$Q=$ rate of discharge

Eigure 2.1 is a diagram of this system.

For long pumping times, when $t>b^{2} s_{s} / 2 K_{z}$, the expression for $f$ may be simplified:

$$
\begin{array}{r}
f=\frac{4 b}{\pi(1-a)} \sum_{n=1}^{\infty} \frac{1}{n}\left(\sin \frac{n \pi l}{b}-\sin \frac{n \pi d}{b}\right) \cos \frac{n \pi z}{b} \\
k_{0}\left[\frac{n \pi x}{b}\left(k_{z} / K_{r}\right)^{1 / 2}\right]
\end{array}
$$




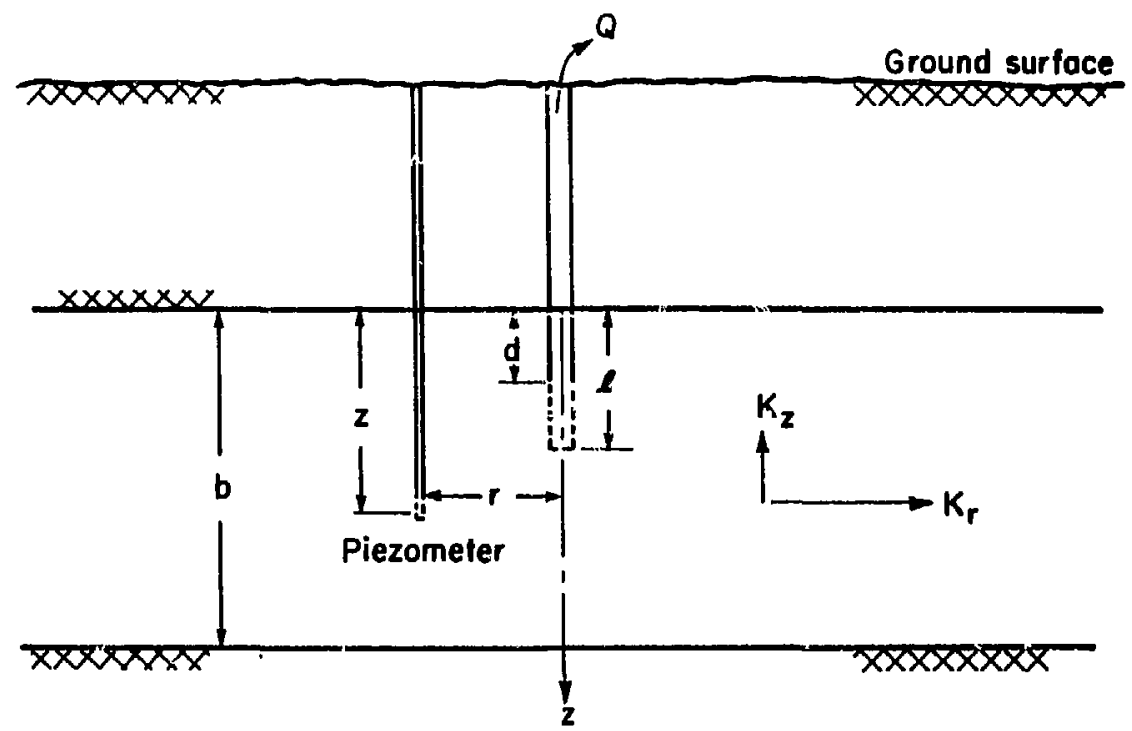

XBL 826-838A

Fig. 2.1. Diagram of a pumping well partially penetrating an anisotropic aquifer with no change of conductivity in radial direction. 
where $k_{0}$ is the modified Bessel function of the second kind and zero order. Comparison of Eq. (2-4) with the analgous equation for an isotropic aquifer shows that, at a distance $I$ from the pumped well, the drawdown due to pumping a partially penetrating well in an anisotropic aquifer is the same as the drawdown at the distance $x\left(x_{2} / x_{1}\right)^{1 / 2}$ in an isotropic aquifer.

The drawdown, s', oue to a fully penetrating well in the anisotropic aquifer is given by:

$$
s^{\prime}=\frac{Q}{4 B R_{r}} \omega\left(u_{r}\right)
$$

This squation shows that the drawdown due to a pumping well which fully perietrates an anisotropic mquifer is controlled by radial hydraulic conductivity and is independent of the vertical hydraulic conductivity.

Depending on the value of $z$, at wich the arawdown is measured in the aquifer (Fig. 2.1), the drawdown aue to a partially penetrating well may be different than that of a fully penetrating well. Weeks uses this difference to obtain the ratio between the vertical and horizontal conductivities of the aquifer. The difference is equal to the difference between Eqs. $(2-1)$ and $(2-5)$

$$
\Delta s=s^{\prime}=\frac{Q f}{4 \pi b k_{r}}
$$

As becomes larger when the vertical hydraulic conductivity is less than the horizontal conductivity. Witherspoon et al. (1967) give a table of the values of $s$ for a large range of parameters. A table of values of $\Delta s$ for different values of $I$ and $z$ is given by weeks (1969). Both of these tables assume isotropic aquifers where $K_{z}=K_{r}$. They can be used for anisotropic cases by substituting $I \sqrt{k_{2} / K_{I}}$ for $r$. Recently, Parr et al. (1983) presented 
programs for HP 41C and TI 59 programable calculators for determining the ratio of horizontal to vertical hydraulic conductivity in an anisotropic aquifer.

Weeks (1969) introduced three methods for analyzing drawdown data to estimate the horizontal to vertical hydraulic conductivity ratio. The choice of method depends on the number of piezometers used in the test. The methods rely upon a comparison of the measured drawdowns in the piezometers to those predicted for a fully penetrating well. The following steps show the general procedure, modified from Weeks:

1. Calculate the radial conductivity of the aquifer, $k_{Y}$, by analyring the test data from an observation well which is open throughout the total thickness of the aquifer. The Theis method may be applied no matter how close the observation weli is to the pumping well.

2. Compute the drawdown value of $s^{\prime}$ at the distance $r$ of the piezometer from the pumping well.

3. Calculate dimensionless correction factor

$$
f=\frac{4_{\pi} b K_{r} s}{Q}
$$

4. From the table of variation of $f$ versus $(x, z)$ (weeks, 1969) find the distance $r_{c}$ at which the drawdown observed in the piezoneter at distance $r$ from the well would have occurred in an isotropic aquifer.

5. Compute the hydraulic conductivity ratio, $\mathrm{k}_{\mathrm{r}} / \mathrm{K}_{\mathrm{z}}$, from:

$$
\frac{k_{r}}{K_{z}}=\left(\frac{r}{r_{c}}\right)^{2}
$$

\section{2: 1.2 Shao-Mokee's Method}

Weeks' method assumes that the horizontal hydraulic conductivity is independent of direction. Shoa-Mckee's method, however, enables one to determine the full hydraulic conductivity tensor of the aquifer assuming that 
two of the principal directions are horizontal and lie on the bedding plane, while the third coincides with the z-axis and is perpendicular to the bedding plane.

Figure 2.2 illustrates the test setup in an anisotropic aquifer satisfying the above constraints. The aquifer is pumped through a partiuily penetrating well at a constant rate of $Q$. The drawdown at a point $(r, z)$ around such a well may ba given by:

$$
\begin{aligned}
& s=\frac{Q}{4 \pi b k_{r}}\left\{w\left(u_{x y}\right)+\frac{2 b}{\pi(1-d)} \sum_{n=1}^{B} \frac{1}{n}\left(\sin \frac{n \pi l}{b}-\sin \frac{n \pi d}{b}\right) \cos \frac{n \pi z}{b} .\right. \\
& \left.w\left(U_{x y^{\prime}} \frac{n \pi x}{b} \sqrt{K_{z} / K_{r}}\right)\right\}
\end{aligned}
$$

This equation is the same as the one used by Weeks, except for the terms:

$$
\begin{aligned}
& \mathrm{k}_{I}=\left(\mathrm{k}_{\mathrm{Xx}} \mathrm{k}_{\mathrm{YY}}-\mathrm{k}_{\mathrm{xY}}^{2}\right)^{1 / 2} \\
& U_{x y}=\frac{S}{4 t} \frac{T_{x x} y^{2}+T_{y y^{x}-2} T_{x y} x y}{T_{x x} T_{y y}-T_{x y}^{2}}
\end{aligned}
$$

where

$$
\begin{aligned}
& T_{X X X}=b k_{X X X} \\
& T_{Y Y}=b k_{Y Y}
\end{aligned}
$$

and $\quad \mathrm{T}_{2 z}=\mathrm{bK}_{2 z}$

Shao-Mckee's method requires a minimum of three piezometers placed near the puming well. Each piezoneter well must be located in a different direction Eran the punping well. The piezometers should be located close enough to the pumping well that $r<b\left(K_{r} / K_{z}\right)^{1 / 2}$ so that the effect of partial Fenetration wil be clearly manifested in the piezoneter measurements. 


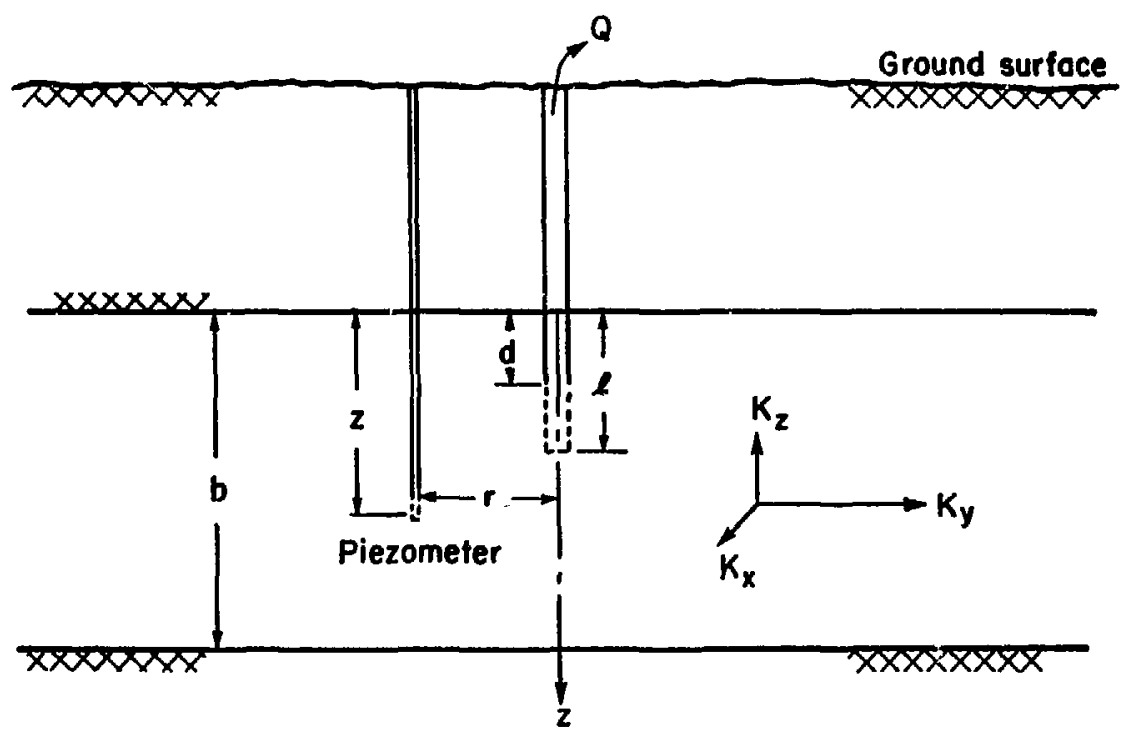

XBL. 828-8388

Fig. 2.2 Diagram of a punping well partially penetrating an anisotropic aquifer where conductivity changes with the direction in the bedding plane. 
The Shao-HcKee method for analyzing field test data is outlined below.

1. Knowing the position of each piezometer $(r, z)$, the screened intarval of the pumping well (1-d), and the thickness of the aquifer (b), prepare a farily of logrlog type curves showing variations of $s_{D}=4 \pi k_{r} b s / Q$ versus $1 / u_{x y}$ with $K_{r} / K_{2}$ as the ruming parameter. These values can be either calculated from Eq - (2-9) or extracted from tables in witherspoon et al. (1967) and Weeks (1969).

2. For each piezometer, plot values of drawdown, $s$, against time, $t$, to the same log-log scale as the type curves.

3. Using superposition, find the best match between the observed data and the type curves. Read the value of $x_{r} / x_{2}$ from the matching type curve and the coordinates of the match point on both graphs.

4. With the value of $s_{D}, s, b$, and $Q$, calculate $K_{r}$ from

$$
K_{I}=\frac{Q s_{D}}{4 \pi b s}
$$

5. Repeat Step 4 for the other two piezometers and caiculate the corresponding $K_{r}$ values. The three valies of $K_{Y}$ obtained with the data from the differen'c piezometers should be approximately equal. If they are not, find an average value of $x_{r}$.

6. Substitute the values of $1 / u_{x y}$ and + from each match point ari the

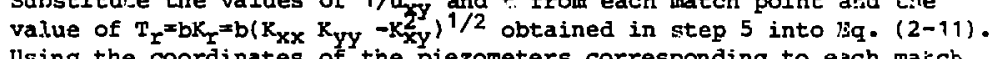
Using the coordinates of the piezometers corresponding to each match point, solve the resulting three equations for the products $S T_{X X}$, $\mathrm{SI}_{\mathrm{YY}}$, and $\mathrm{ST}_{\mathrm{XY}}$.

7. Cajculate the value of $s$ fron

$$
\mathrm{S}=\frac{\left[S T_{\mathrm{xx}} S T_{\mathrm{yy}}-\left(S T_{\mathrm{xy}}\right)^{2}\right]^{1 / 2}}{b \mathrm{x}_{\mathrm{r}}}
$$

8. Having found $s$, calculate $T_{x x}, T_{y y}$, and $T_{x y}$ and consequentiy, $K_{x x}$, $K_{Y y^{\prime}}$ and $K_{x y}$ from the products obtained in step 6 .

9. Calculate $k_{z}$ by dividing $k_{r}$, obtained from step 5 , by $k_{r} / k_{z}$ obtiuned in Step 3 . 


\subsection{Tests gased on the Theory of Leaky Aguifers}

A "leaky aquifer" is an aquifer that is over- and/or underlain by much less permeable layers. When the pressure in the aquifer is reduced by pumping, water from the saturated, less permeable layers above or below leak into the aquifer. Sometimes, the leakage is so great that it can be detected in the aquifer being pumped. In this case, the confining beds are called "aquitards" and the aquifer is said to be be "leaky." When the leakage is so little that it cannot be easily detected, the confining beds are called "aquicludes" and the aquifer is termed "slightly leaky" (Neuman and Witherspoon, 1968).

Much work has been done on the theory of leaky aquifers. The first papers appeared before 1960 (Jacob, 1946; Hantush and Jacob, 1955; and Hantush, 1956) and were based on the assumption that the storage capacity of aquitards or aquicludes was negligible. In 1960, Hantush introduced a theory of leaky aquifers which considered the effect of storage capacity of the confining bed. Neuman and Witherspoon (1969, 1972) evaluated the significance of Hantush's assumptions and provided moxe generalized solutions. All of the leaky aquifer solutions assume that flow in the less permeable layer, above or below an aquifer, is essentially vertical. Therefore, these methods permit the average vertical hydraulic conductivity for the confining layer to be estimated.

\subsubsection{Hantush and Jacob Solution}

Jacob (1946) developed a partial differential equation for a lesky aquifer and solved it for a bounded reservoir. Hantush and Jacob (1955) solved the same problem for a radially infinite aquifer. Although these 
solutions are not applicable in all cases, they are widely used by groundwater hydrologists because of their simplicity.

Figure 2.3 depicts a leaky aquifer system. A semi-permeable layer (aquitard) with a constant thickness of b' overlies an aquifer with much higher hydraulic conductivity. The aquitard is overlain by another highly permeable and extensive aquifer. The lower aquifer is pumped with a constant rate of discharge Q. Hantush and Jacob (1955) obtained an expression which gives the drawdown distribution in the pumped aquifer as a function of time. Derivation of this solution was based on three major assumptions: (1) flow is essentially horizontal in the aquifer and vertical in the aquitard, (2) no drawdown occurs in the upper aquifer due to pumping in the lower aquifer, (3) leakage into the pumped aquifer is proportional to the potential drop across the aquitard. This last assumption is equivalent to assuming that the storage capacity of the confining bed is negligible, that all the water leaking into the pumped aquifer comes directly from the upper aquifer, and that the aquitard brthaves only as a conduit between the two aquifers. The solution to this problem, sometimes referred to as the 'r/B solution', is

$$
s=\frac{Q}{4 \pi K b} w(u, r / B)
$$

where

$$
\begin{aligned}
& u=\frac{r^{2} s}{4 t K} \\
& \mathrm{~B}=\sqrt{\frac{K \mathrm{~K} b^{\prime}}{\mathrm{K}^{\prime}}} \text { called the leakage factor; } \\
& K^{\prime} K^{\prime}=\text { hydraulic conductivity of the aquifer and aquitard, respectively } \\
& s_{s}=\text { specific storage }
\end{aligned}
$$




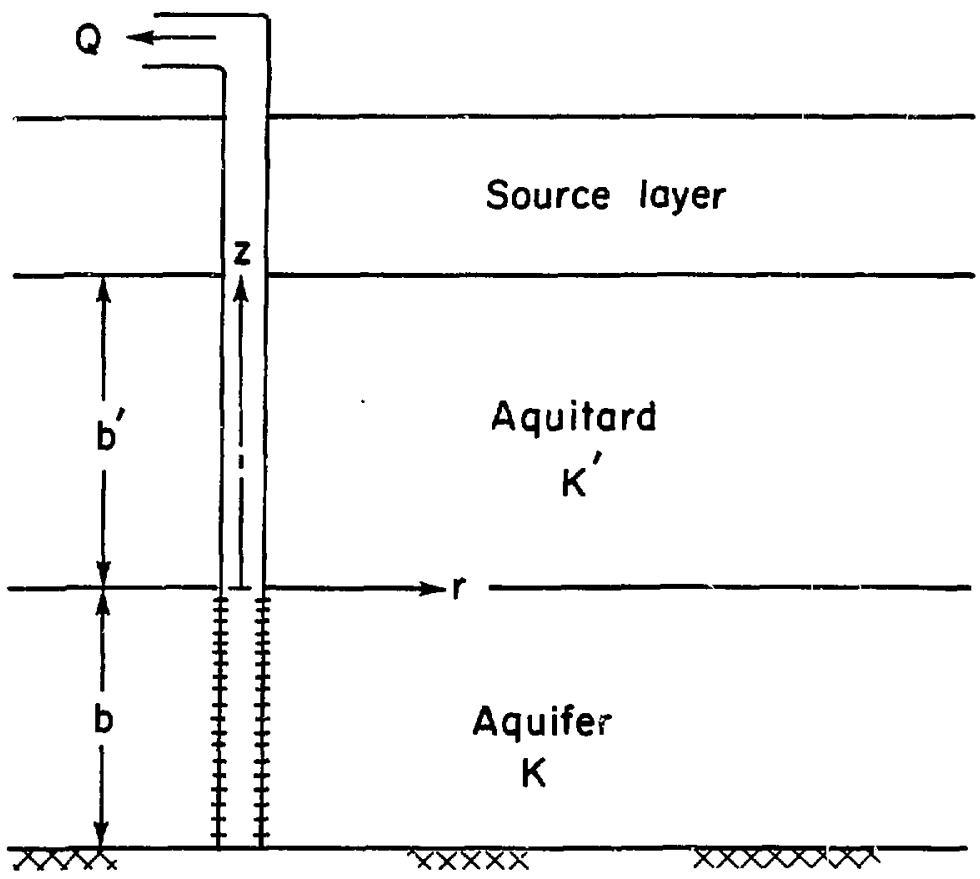

XBL 826-842

Fig. 2.3 Diagram of a leaky aquifer pumped by a fully penetrating well. 


$$
\begin{aligned}
& s \\
& b, b^{\prime}=\text { drawdown in the aquifer } \\
& W(L, r / B)=\int_{u}^{\infty} \exp \left(-y-\frac{r^{2}}{4 y B^{2}}\right) \frac{d y}{y}
\end{aligned}
$$

This last term is the well function of leaky aquifers, which has been extensively tabulated (Hantush, 1956).

The procedure for conducting a leaky aquifer test is similar to that for a standard pump test in a simple aquifer. From such a test one obtains a table of drawdown in an observation well (or piezometer) against time elapsed fron the start of pumping.

Several methods based on the $r / B$ soilution are corventionally used for interpretation of leaky aquifer pump test data. A. Walton's Type-Curve Method (1960)

- Prepare a family of type curves by log-log plorting the values of function $W(u, r / B)$ versus $1 / u$ with $r / B$ as the running parameter of the curves. The curve with $r / B=0$ is the Theis curve.

- With the same scale used for the type curves, make a log-log plot of the drawdowns versus time as recorded in an observation well.

- Match the curves and find the value of $t / B$ by interpolating the position of the data curve among the type curves. Also find the dual coordinates of the matching points, $s, t, 1 / u$, and $w(u, r / B)$. A unique fitting position is difficult to obtain unless sufficient data is available from the period when the leakage erfect is insignificant (Hantush, 1964).

- Calculate the hydraulic conductivity of the pumped aquifer from

$$
K=\frac{Q}{4 \pi b s} W(u, r / B)
$$

- Calculate the specific storage of the pumped aquifer from

$$
s_{s}=\frac{4 t K}{r^{2}(1 / u)}
$$


o Finally, calculate the vertical conductivity of the aquitard from

$$
K^{\prime}=\frac{k b b^{\prime}}{r^{2}}\left(\frac{r}{B}\right)^{2} .
$$

B. U.S.B.R. Method

Fullowing is the method which the U.S. Bureau of Reciamation Groundwater Manual (1977) suggests for interpretation of pump test data of a leaky aquifer. Figure 2.4 showg a family of type curves prepared by the united States Bureau of Reclamation from Jacob's (1946) leaky aquifer solution developed for a radially bounded aquifer. However, in developing Fig. 2.4 the outer boundary was located at a sufficient distance from the pumping well that the head at the boundary did not change due to pumping. (Glover, Moody, and Tapp, 1960). This approach permits the curves to be used for infinite aquifers. The steps for applying the USBR method are:

- Make a log-log plot of drawdown versus time from two or more observation wells (after appropriate corrections) locatea at different radial distances $r$ from the pumped well with the same scale as Fig. 2.4.

- Superimpose the field data curves on those of Figure 2.4.

- After obtaining the best match between the field data and one of the curves on Fig. 2.4, find the dual coordinates of a match point $(s, t, u$ and $n)$, and the $x$ value of the best fitting type curve. Interpolation may be required to find the $x$ value.

- Calculate the hydraulic conductivity of the aquifer from

$$
K=\frac{Q u}{2 \pi M s}
$$

- Calculate the hydraulic conductivity of the aquitard from

$$
K^{\prime}=K M M^{\prime}\left(\frac{x}{x}\right)^{2}
$$




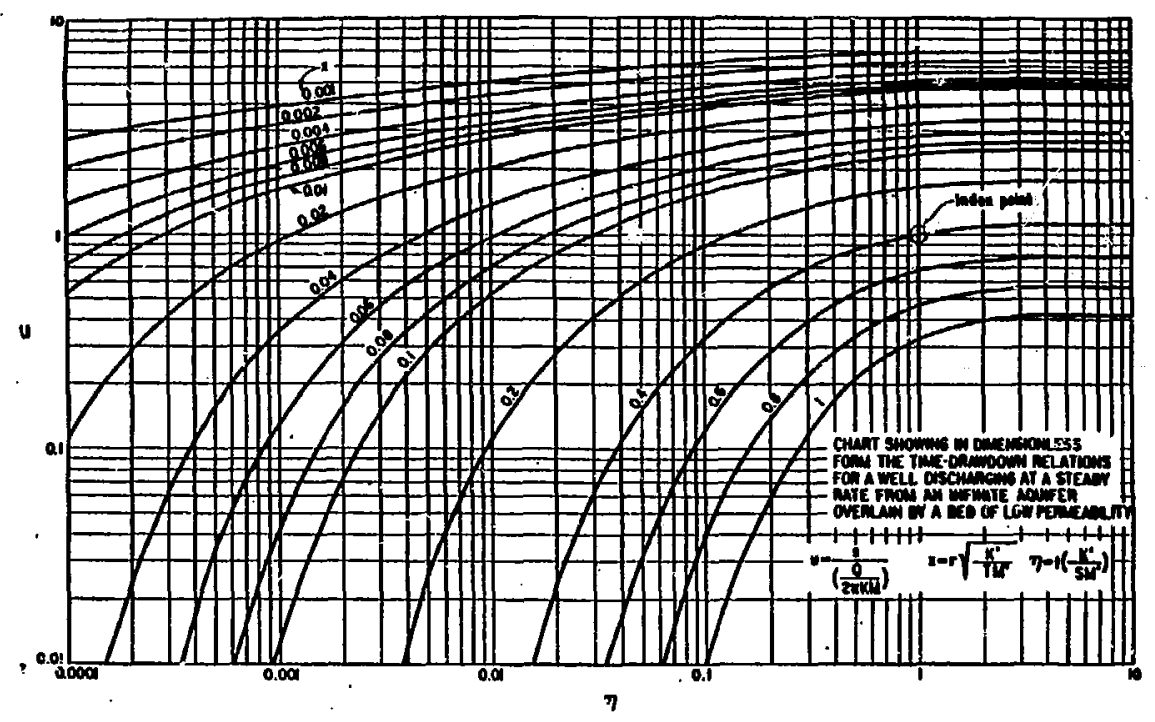

Fig. 2.4 Leaky aquifer type curves based on $r / B$ solution (USBR, 1977) 
- Finally, calculate the storage coefficient of the aquifex from

$$
s=\frac{R^{\prime} t}{n M}
$$

In the above equations, $M$ and $M$ ' iridicate the thickness of the aquifer and the aquitard, respectively. The ratio $r / x$ is the leakage factor $B$ used in the development of the theory. The definitions of other terms are given in Fig. 2.4. Other methods of analysis of field data based on $r / B$ solution have been suggested by Hantush (1964, p. 416-417), and Narasimhan $(1968)$.

Uncertainties in the Interpretation of Leaky Aquifer Tests by the Hantush and Jacob solution

The problem of flow to a pumped well in a hydrolugic system consisting of several aquifers separated by less pervious aquitards or aquicludes is in fact 3 dimensional. A rigorous approach to the solution of such a problem is analytically intractatile. Therefore, it has been sustomary to simplify the problem by assuming that flow is essentially horizontal in the aquifers an 3 vertical in the aquitards and aquicludes. Neunan and Witherspoon (1969) evaluated the validity of this assumption which was used in the derivation of the $r / B$ solution. They noted that the errors it introduces are less than 58 if the conductivities of the aquifers are more than 3 orders of magnitude greater than that of the aquitards. The errors increase with time and decrease with radial distance from the pumping well. One should note that the 58 error described by Neuman and witherspoon (1969) is the percentage difference between drawdowns calculated with an analytic solution based on the standard assumptions and the drawdowns calculated with a finiteelement numerical analysis which does not require these assumptions. The magnitude of the error which may result in the calculation of the hydraulic 
properties of the confining layar is not known.

Another assumption used in the derivation of the $r / B$ solution is that no water is released from storage in the aquitard. Neuman and witherspoon (1969) found that this assumption tends to result in overestimating the permeability of the aquifer and underestimating the permeability of the iquitard.

Another important uncertainty in the $r / B$ solution is that it does not distinguish between groundwater leakage from the strata above the pumped aquifer, below the pumped aquifer or soie combination thereof. This uncertainty is particularly important when the purpose of the test is to assess the hydraulic concuctivity of one of the confining strata rather than that of the aquifer itself.

When the hydraulic conductivity of the confining bed is so small that the ratio of $K^{\prime} / K$ tends to zero, the drawdown distrilution in the aquifer becomes essentially the same as would be predicted by the Theis solution for an aquifer without leakage. As a result, techniques based on observation in the aquifer alone fail to give the properties of the confining bed.

\subsubsection{Hantush Modified Solution}

In 1360 Hantush intrcduced a treatment of leaky aquifers which overcame some of the difficulties of the $r / B$ solution. In iddition to assigning a storage capacity to the confining aquitard, he solved the problem for (1) an infinite horizontal aquifer overlain by an aquitard whose upper boundary does not experience any change in head from pumping the aqufer and (2) the same situation but with an impermeable bed overlying the aquitard. 
Other assumptiong applied in the development of the $r / B$ golution, including vertical flow in the aguitard and horizontal flow in the aquifer, still hold. In this solution Hantush considsred leakage tnto the aquifer from both above and below. He presented the solutions for two ranges of tive, $t$.

\section{Solutions for Small Values of Time}

For $t$ less than both $b^{\prime} s^{\prime} / 10 \mathrm{~K}$ ' and $\mathrm{b}^{\prime \prime} \mathrm{s}^{\prime \prime} / 10 \mathrm{~K}$, the solution for both cases ( 1 ) and (2), mentioned above, is the same:

$$
g=\frac{\Omega}{4 \pi K b} H(u, \beta)
$$

where

$$
\begin{aligned}
& H(u, B)=\int_{u}^{\infty} \frac{e^{-y}}{y} \operatorname{erfc}(\beta \sqrt{u} / \sqrt{y(y-u)}) d y \\
& B=(F \lambda) / 4 \\
& \lambda=\sqrt{\frac{\mathrm{K}^{\prime}}{\mathrm{Kbb^{ \prime }}} \frac{\mathrm{S}^{\prime}}{\mathrm{S}}}+\sqrt{\frac{\mathrm{K}^{n}}{\mathrm{~Kb}^{n}} \frac{\mathrm{S}^{n}}{\mathrm{~S}}} \\
& v=\frac{r^{2} s}{4 t b k} \\
& s \text { = drawdown in the aquifer } \\
& S^{\prime \prime} . S^{\prime}=\text { storage coefficient of the lower and upper aquitard, } \\
& \text { respectively } \\
& K^{\prime \prime}, K^{\prime}=\text { hydraulic conductivity of the lower and upper aquitards, } \\
& \text { respectively } \\
& \text { I * radia, distance of the observation well from the pumped } \\
& \text { well } \\
& b^{\prime \prime}, b^{\prime}=\text { thickness of aquitards below and above the aquifer, } \\
& \text { respectively. }
\end{aligned}
$$

An extensive table of $H(u, \beta)$ is in Hantush (1960b); a short table is available in Hantush (1964). 
Solution for Large Values of Time

Case 1.

In this case, $t$ should be larger than both $5 b^{\prime} s^{\prime} / K^{\prime}$ and $5 b^{\prime \prime} s^{n} / K^{\prime \prime}$. The solution is then:

$$
s=\frac{Q}{4 \pi b K} w\left(u \delta_{1}, a\right)
$$

where

$$
W\left(u \delta_{1}, \alpha\right)=\int_{u \delta_{1}}^{\infty} \frac{d y}{y} \exp \left(-y-\frac{\alpha^{2}}{4 y}\right)
$$

is the well function for leaky aquifers tabulated by Hantush (1956);

$$
\begin{aligned}
& a=I \sqrt{\frac{K^{\prime}}{b b^{\prime} K}+\frac{K^{\prime \prime}}{b b^{\prime \prime}}} \\
& \delta_{1}=1+\frac{s^{\prime}+s^{\prime \prime}}{3 s}
\end{aligned}
$$

The other terms are the same as defined for small values of time.

\section{Case 2.}

For $t$ greater than both $10 \mathrm{~b}^{\prime} \mathrm{S}^{\prime} / \mathrm{K}^{\prime}$ and $10 \mathrm{~b}^{\prime \prime} \mathrm{S}^{\prime \prime} / \mathrm{K}^{\prime \prime}$ the expression for drawdown in the aquifer is

$$
s=\frac{Q}{4 \pi \mathrm{Kb}} W\left(\mathrm{u} \delta_{2}\right)
$$

where

$$
\begin{aligned}
W\left(u \delta_{2}\right) & =\int_{u \delta_{2}}^{\infty} \frac{e^{y}}{y} d y \text { is the well Eunction } \\
\delta_{2} & =1+\frac{s^{\prime}+s^{\prime \prime}}{s}
\end{aligned}
$$

The choice of solution depends upon the hydrolugic properties of the system being tested and the curation of pumping. For large values of time, 
Eq. (2-32) indicates that, even when one considers the storage capacity of the confining bed, the $x / B$ solution could be safely used for evaluation of the aquifer and aquitard, provided that

$$
t>\frac{5 b^{\prime} s^{\prime}}{x^{\prime}}
$$

This solution may also qualify at small times when the aquitard is thin, when it has a relatively high hydraulic conductivity, and is incompressible (i.e., very small $\left.\mathrm{s}^{\prime}\right)$. For example, if $\mathrm{b}^{\prime}=5 \mathrm{~m}, \mathrm{~K}^{\prime}=2 \times 10^{-7} \mathrm{~m} / \mathrm{s}$, and $\mathrm{s}^{\prime}=2 \times 10^{-5} \mathrm{~m}^{-1}$, then the $r / B$ solution is applicable after 625 seconds (approximately 10.5 minutes) after the start of the test if $u$ in Eq. $(2-17)$ is replaced by

$$
u\left(1+\frac{s^{\prime}}{3 s}\right)
$$

For the solution to apply, the aquifer above the aquitard should not show any drawdown during the test. If the overlying aquifer does show drawdown then the $r / B$ solution tends to underestimate the hydraulic conductivity of the aquitard. However, if the confining bed is relatively thick and elastic with low hydraulic conductivity then the $I / B$ solution is not applicable. For example, if $b^{\prime}=50 \mathrm{~m}, \mathrm{~K}^{\prime}=5 \times 10^{-9} \mathrm{~m} / \mathrm{s}$ and $\mathrm{s}^{\prime}=10^{-3}$, then the $\mathrm{r} / \mathrm{B}$ solution is only applicable after $3.12 \times 10^{8}$ seconds, (approximately 1 year) after the test has started.

Equation (2-36) suggests that wheri the confining bed is thin, relatively permeable and incompressible, and overlain by an impermeable layer which cannot supply water, the drawdown data from the aquifer will follow the Theis solution relatively soon after pumping is stated. In applying the Theis solution, $u$ in the well function should be replaced Ly

$$
u\left(1+\frac{s^{\prime}}{s}\right) \text {. }
$$


Although Eq: (2-27) is for early-time, it can be applied to later times when the aquitard is thick, relatively impermeable, and compressible. For example, if $b^{\prime}=100 \mathrm{~m}, K^{\prime}=10^{-9} \mathrm{~m} / \mathrm{s}$, and $s^{\prime}=10^{-3}$, then $\mathrm{Eq} \cdot(2-27)$ is applicable for $10^{7}$ soconds or the first 115 days, of the test. Within this time range, the effect of pumping will not reach the upper boundary of the aquitard. Therefore, the assumption of a constant head boundary there does not introduce any error. Although our discussion only refered to the upper confining bed, both the upper and lower beds must meet the same criteria for these simplifications to apply.

The test procedure is the same as for a standard pump test. The data needed for interpretation is a rrsord of drawdown versus tims in one or more observation wells in the aquifer around a pumping well.

Eigure 2.5 shows a family of type curves on a log-log plot of $\mathrm{H}(\mathrm{u}, \beta)$ versus 1/u. The plot can be used for the Hantsh Modified Solution analysis. The procedure for analyzing the pump test data is outlined below.

- Make a log-log plot of the variation of drawdown versus time with the same scale as that of the type curves.

- Superpose the observed plot and the appropriate type curve to find the best match.

- Read the value of $B$ from the type curve which matches the observed plot, and the dual coordinates $H(u, \beta), 1 / u, t$, and $s$ of the match point.

- Calculate the hydraulic conductivity of the aquifer from

$$
K=\frac{Q}{4 \pi b s} H(u, B)
$$

- Calculate the storage coefficient of the aquifer from

$$
s=\frac{4 t b K u}{r^{2}}
$$




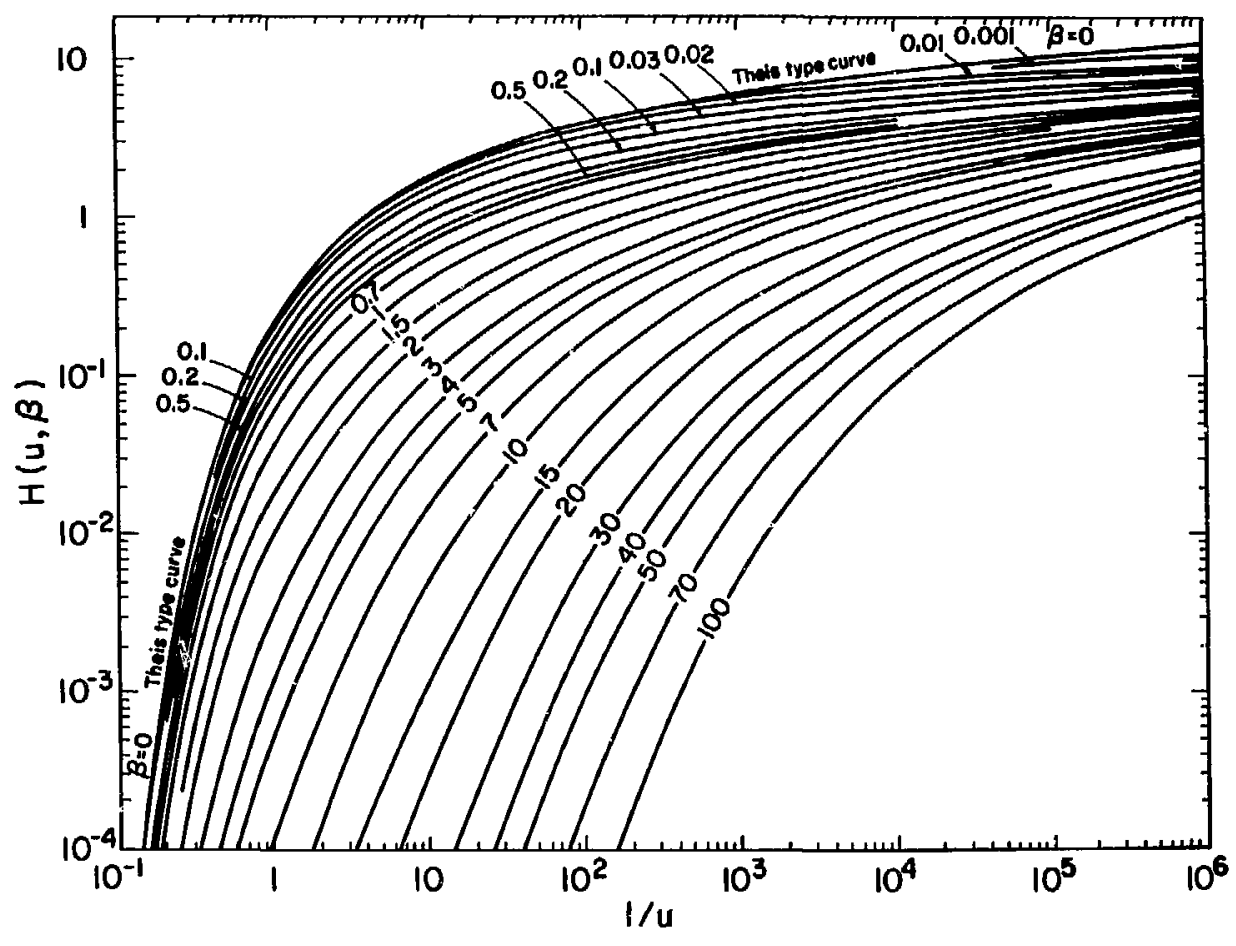

Fig. 2.5 Type curves of the function $H(u, B)$ versus $1 / u$, for various values of B (after Lohman, 1972). 
- Calculate $\lambda$ from

$$
\lambda=\frac{48}{r}
$$

- If we assume that the lower layer is completely impermeable, then

$$
\mathrm{k}^{\prime} \mathbf{s}^{\prime}=\lambda^{2} \mathrm{Kbb}^{\prime} \mathbf{s}
$$

- If one can determine the magnitude of the storage coefficient of the aquitards, irom other methods, then the hydraulic conductivity of the aquitard may be obtained from

$$
K^{\prime}=\frac{\lambda^{2} x b b^{\prime} s}{s^{\prime}} .
$$

Uncertainties in Interpretation of Ieaky Aquifer Tests by the hantush Mocified Solution

Except for very large values of $\beta$, the type curves have very similar shapes that are not much different from the Theis curve. Thus, it is difficult to decide which of the type curves to use in matching against field data. When $B$ is very small, it is easy to make an error of ds much as two orders of magnitude when selecting a value for $B$. Because

$$
K^{\prime} S^{\prime}=\left(B^{2}\right) \frac{16 K b b^{\prime} S}{r^{2}}
$$

an error in choosing $\beta$ leads to much larger error in the calculation of (K'S'). Thus, a two orders of magnitude error in estimating $B$ would lead to foux orders of magnitude error in ( $\mathrm{K}^{\prime} \mathrm{s}$ ').

To ameliorate this problem, Weeks (1977) suggested using datd from at least two observation wells at different distances from the pumping well. A composite $\log -\log$ plot oE the drawdown versus $t / x^{2}$ is made with the same scale as that of the type curves. In this way two or more type curves, each with different values of $B$ proportional to the value of $r$, can usually be 
obtained. A unique match may then be obtained by adding the extra constraint that $r$ values for observation wells must fall on curves having proportional $p$ values (Weeks, 1977). This method can somewhat reduce the uncertainties in the data interpretation but, when $\beta<0.01$, type curves with differenc values for $B$ are so close together that a unique match is still almost impossible to identify.

In many cases, groundwater from both the strata above and below an aquifer leaks into the aquifer. In these circumstances it may not be pos. sible tc determine the hydrologic properties of either of the confining layers. This is because the Hantush Modified solution yields only the value of $\lambda(\mathrm{Eq}, 2-42)$, which is a parameter depending on the properties of both confining layers and the aquifer. Thus, the method provides no means for indepenently determining the properties of the confining layers.

In cases where leakage into the aquifer is from only one of the confining layers, the method gives the product of the hydraulic conductivity and the storage coefficient of the aquitard. The value of the storage coefficient for the aquitard is not determined independently and must be found by some other means before one can finally obtain the vertical hydraulic conductivity.

\subsubsection{Witherspoon and Neuman Ratio Method}

When the ratio of $K^{\prime} / K$ decreases, both $r / B$ and $\beta$ decrease and Eqs. (2-17) and (2-27) will eventually reduce to the Theis solution and determination of the hydraulic conductivity of a tight confining layer by observing drawdown in the aquifer alone will be difficult or impossible. Witherspoon et al. (1962) suggested a method of calculating the permeability of the 
caprock of gas storage reservoirs using observations of drawdown in both the aquifer and the overlying aquitard. Witherspoon and Neuman (1967) improved the method and further refined the technique (Neuman and witherspoon 1972). The theory is summarized below.

Consider an aquifer of finite thickness overlain by a semi-infinite confining bed. When the ratio of $K^{\prime} / K$ is sufficientily small, then under the influence of pumping the aquifer, the flow in the confining bed is essentially vertical, and the drawdown in the aquifer can be ciosely approximated by the Theis solution. The term "semi-infinite" indicates that the aquiclude is so thick that the effect of pumping the aquifer does not reach the top of the aquiclude. With these assumptions Witherspoon and Neuman (1967) derived an expression which gives the drawdown in the aguiclude as a function of time, $t$, and elevation, $z$, ebove the top of the aquifer:

$$
s^{\prime}=\frac{Q}{2 \pi^{3 / 2} k b} \int^{\infty} \frac{t^{1 / 4 t^{\prime}}}{D}-E i\left[-\frac{t_{D}^{\prime} y^{2}}{t_{D}\left(4 t_{D}^{\prime} y^{2}-1\right)}\right] \bar{e}^{2} d y
$$

where

$$
\begin{aligned}
t_{D}^{\prime} & =\frac{x^{\prime} t}{s_{s}^{\prime} z^{2}} \\
t_{D} & =\frac{K t}{s_{s} x^{2}} \\
-E i(-x) & =\int_{x}^{\infty} \frac{\bar{e}^{Y}}{y} d y
\end{aligned}
$$

$z \quad=$ vertical distance from the top of the aquifer

$S_{S^{\prime}} S_{S}^{\prime}=$ specific storage of the aquifer and the aquiclude, respectivelv. 
Figure 2.6 shows a family of curves presenting variation of $\mathrm{s}^{\mathrm{H}} / \mathrm{s}$ versus $t_{D}$ for different values of $t_{D}$. Drawdown in the aquifer is assigned the variable name $s$.

Neuman (1966) also solved a variation of the above problem ror a finite thickness aquiclude. In this derivation the hydraulic head was assumed to be constant at the top of the aquiclude. This solution has been evaluated over a practical range of relevant dimensionless parameters and the results are tabulated in Appendix $\mathrm{H}$ of Witherspoon et al. (1967).

Neuman and Witherspoon (1969a) developed a complete solution for the distribution of drawdown in a system consisting of an aquitard that separates two aquifers (Fig. 2-7). In each aquifer the solution depends on five dimensionless parameters; in the aquitard, six dimensioniess parameters. Consequently, Neuman and witherspoon (1972) stated that "This large number of digensiorless parameters makes it practically impossible to construct a sufficient number of type curves to cover the entire range of values necessary for field application." Hantush (1960) apparently had noticed this problen earlier as he stated that "It should be remarked that rigorous solutions can be obtained for the actual nonsteady three-dimensional flow $2 \mathrm{n}$ layered aquifers, as well as solutions for flow systems in which the condition of vertical leakage is removed. These solutions, however, are very difficult to evaluate numerically and are therefore not presented here."

Because sophisticated theories for the analysis of test data from leaky aquifer systems are difficult to apply in the field, most authorities recommend the simpler approaches. For example, all the methods described by Hantush (1964) are based on the $r / B$ solution even though they were published 


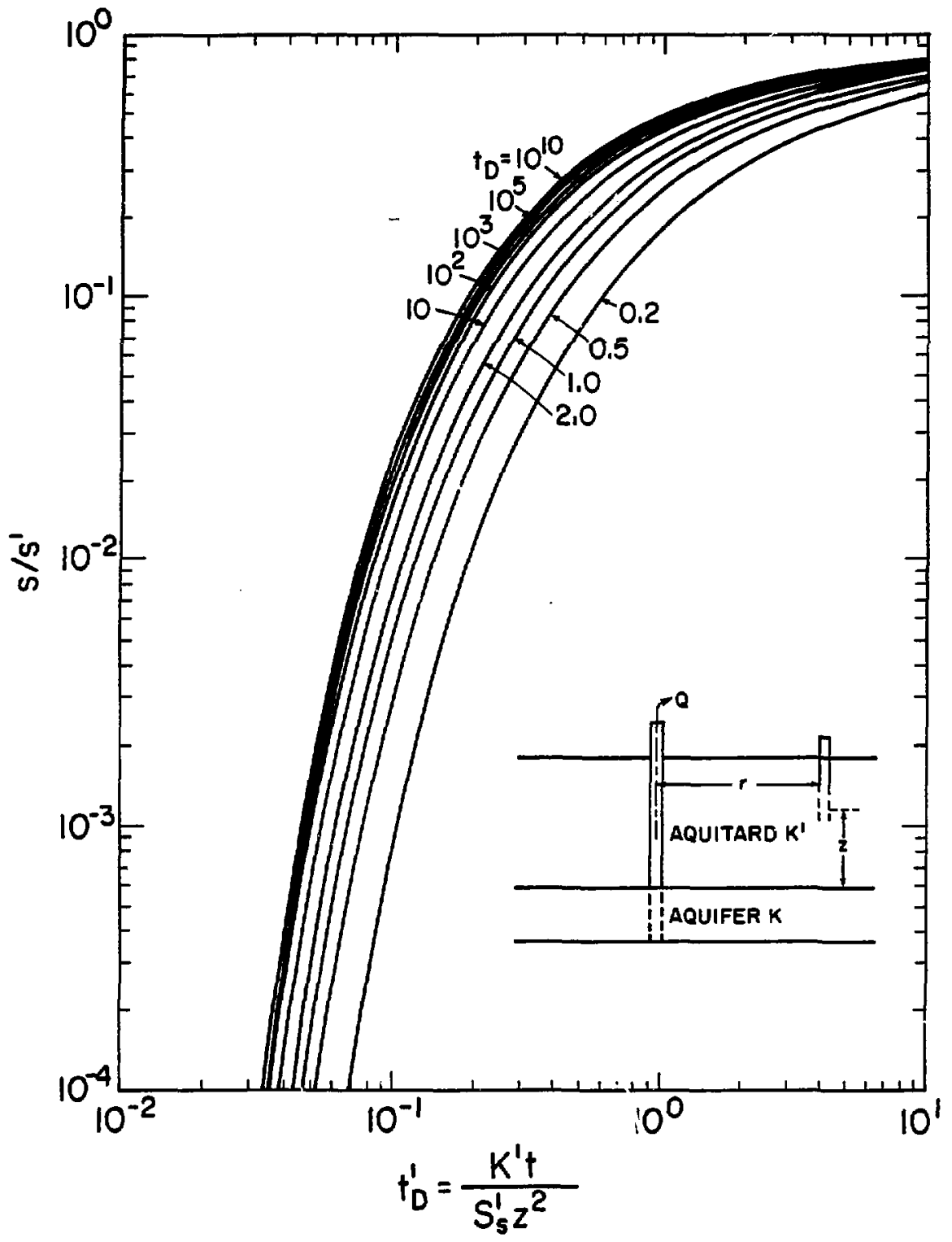

XBL835-1828

Fig. 2.6. The variation of $s^{\prime} / s$ with $t^{\prime}{ }_{D}$ for a sime-infinite aquitard (modified from Witherspoon et al., 1967) 


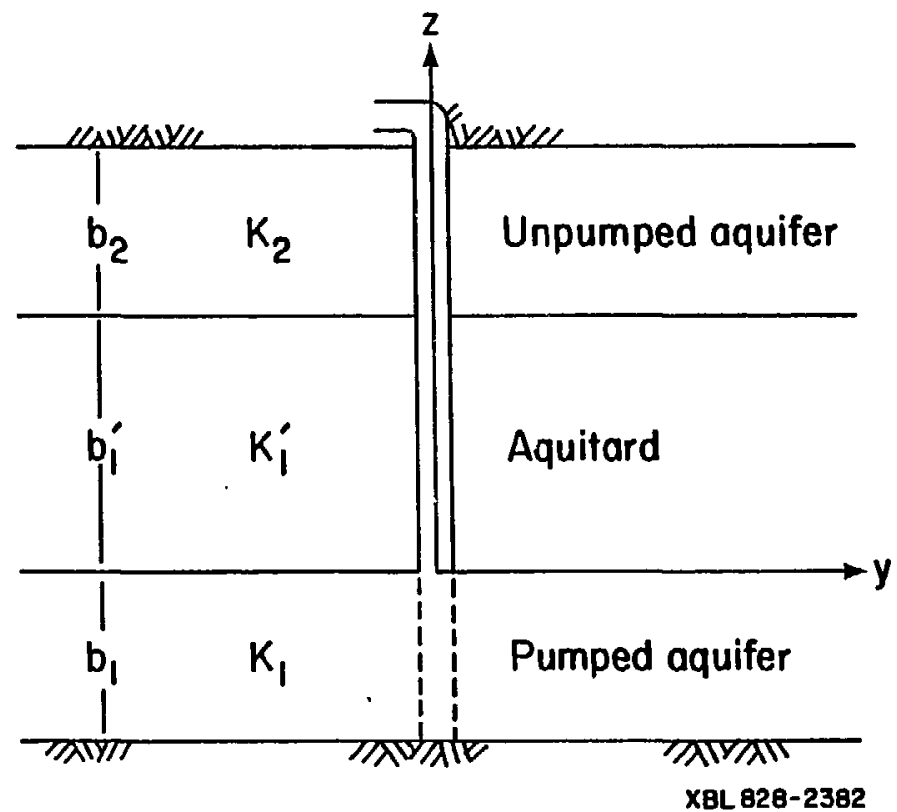


four years after he introduced the modified theory in 1960. In the five years following the original publication of their ratio method in 1967 , Neuman and witherspoon developed a more sophisticated theoretical technique for analysis of leaky aquifer tests but they recognized the difficulties of practical application. In 1972 they stated that "we therefore decided to adopt the ratio method as a standard tool for evaluating the properties of aquitards."

The procedure for performing tests according to the witherspoon and Neuman Ratio Method is summarized below.

- Complete a pumping well through the total thickness of the aquifer.

- Construct an observation well in the aquifer at a distance $r$ from the axis of the pumping well.

- Establish at least three transducers to measure hydraluic head at three different elevations within the confining bed, as shown in Fig. 2.8. Ideally, the piezometers should be nested in the same well. If separate boreholes are used, the radial distance of all three transducers from the pumping well should be the same as that of the observation well. Water pressure should also be measured in the observation well within the aquifer.

- Start recording water pressures long before the start of the pumping test. It is very important that the pressures reach equilibrium before the beginning of the test.

- start producing from the pumping well with a constant rate of $Q$. Pumping should continue until at least half a meter of drawdown is observed by the middle transducer in the aquiclude. Recording of heads should continue at least a few days after pumping has stopped.

The test data is analyzed as follows:

o observe the pressure record of the traneducer at the top of the confining bed. If it shows any drawdown beyond that assumed as a limiting condition for the theoretical solution, note the time of such observation and ignore all records of drawdown measured after that time. 
PUMPING WELL

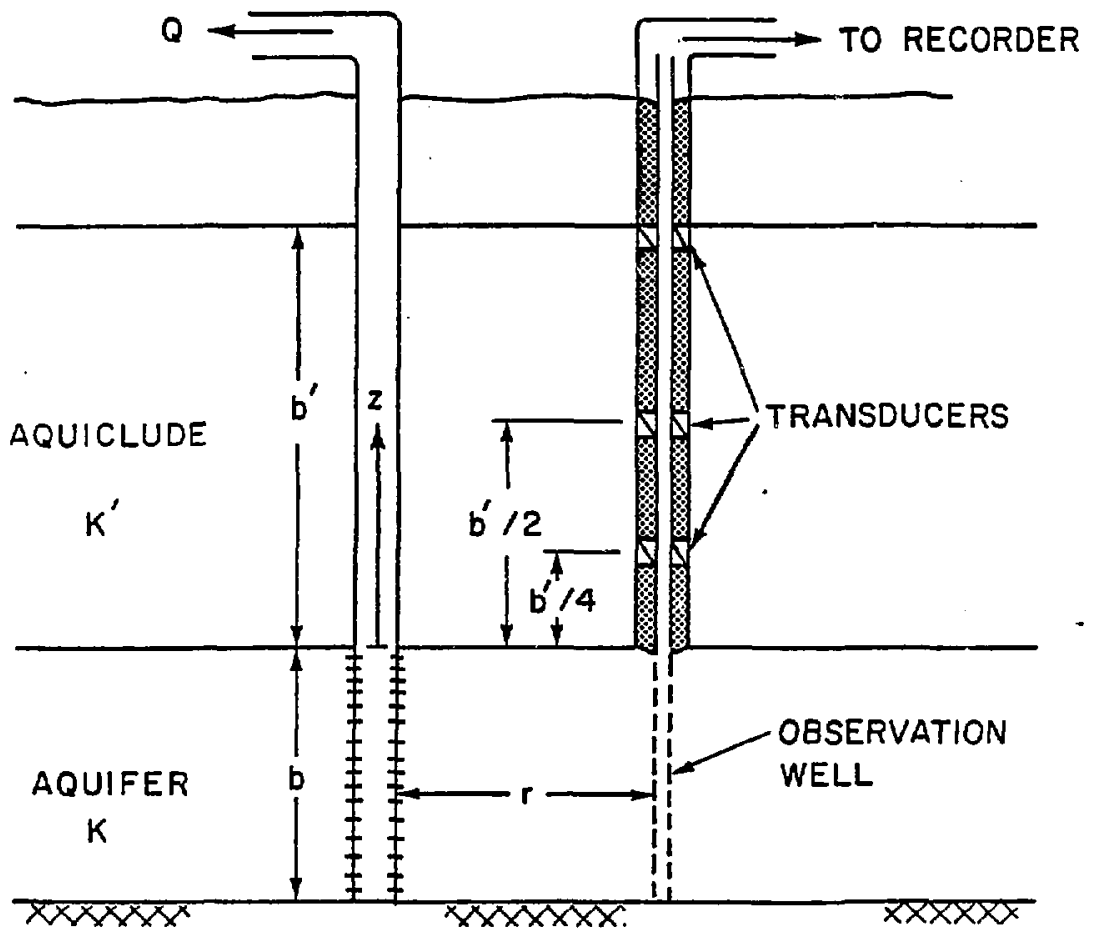

XBL $826-843$

Fig. 2.8 An arrangement of a ratio method test. 
- Calculate the hydraulic conductivity, $\mathbf{K}$, and the specific storage $S_{s}$, of the aquifer with Hantush's modified solution and the drawdown recorda from the observation well.

- Make a log-log plist of the values of drawdown versus time, both in the aquifer and the aquiclude and draw smooth curves through the data.

- Select several arbitrary values of time t. All values should be smaller than the time when drawdown was first noted at the top transducer.

- Calculate $t_{D}$ for each of the selected values of $t$ from the following equation.

$$
t_{D}=\frac{K t}{s r^{2}}
$$

- At each value of time, select representative values of $s$ and $s$ " from the time drawdown plots.

- Using the appropriate curve corresponding to each value of $t_{D}$ from Fig. (2.6), find $t^{\prime} \mathrm{D}$ for each ratio of $\mathrm{s}^{\prime} / \mathrm{s}$.

- Calculate the vertical diffusivity of the confining bed for each value of $t$ and $z$ of a particular transducer from

$$
\frac{K^{\prime}}{s_{s}^{\prime}}=-\frac{z^{2} t_{D}^{\prime}}{t}
$$

- For each value of $z$, find the average value of $K^{\prime} / \mathrm{s}^{\prime} \mathrm{s}$ for different times. The average value calculated for each $z$ should represent the diffusivity of that part of the confining layer between the top of the aquifer and that particular elevation.

Advantages of the Witherspoon and Neuman Ratio Method for Interpretation of Leaky Aquifer Tests

As was noted before, if the aguifer received leakage from both above and below, then $r / B$ and $B$ methods, which rely on the measurement of drawdowns in the aquifer alone, cannot be used to calculate the hydraulic conductivities of the confining layers. The ratio method, on the other hand, can be use to calculate diffusivities of both upper and lower confining beds. 
Uncertainties in Interpretation of Leaky Aguifer Tests by Wharspoon and Ne:man Ratio Methods

Only the vertical diffusivity of the confining beds can be desermined with the ratio method. However, if one can calculate their specific storage by other means, then their vertjcal hydraulic conductivities can also be computed. Leahy (1976) overcame the difficulty by using Hantush's (1960) B solution to find the product of $K^{\prime}$ and $S^{\prime}{ }_{s}$ ' and the witherspoon and Neuman (1967) ratio method to find the ratio of $\mathrm{K} / \mathrm{s}$ 's ' Then, he calculated the value of $K^{\prime}$ from

$$
K^{\prime}=\sqrt{\left(\frac{K^{\prime}}{s_{g}^{\prime}}\right) \cdot\left(K^{\prime} \cdot s_{g}^{\prime}\right)}
$$

The method is based on the assumption that the hydraulic head remains constant at the top of the confining bed. Depending on the thickness and the hydraulic properties of the aquitard, this may cause errors in the result. If the aquitard is thin and has a small storage coefficient, the transient effect may completely penetrate it at relatively early stages of the pump test.

Wolff (1970) reported that piezometers completed in an aquitard exhibited reverse water-level fluctuations; that is, water levels rise for some time after the start of pumping from the aquifer. ile relates these changes to radial and vertical deformation of the aquifer and zquitard resulting from their compressibility. Because the ratio method does not take such phenomena into account, Weeks (1977) warns against the method. However, this phenomenon has not been observed in other tests such as the ones reported by Leahy (1977), and Neuman and Witherspoon (1972). 


\subsection{Tests Based on The Concept of Directional Hydraulic Conductivity}

In fractured media, the principal directions of hydraulic conductivity are usually governed by the fracture configurations. Therefore, the assumption that two of the principal directions of hydraulic conductivity lie on the bedding plane may not hold. If the assumption cannot be made, in addition to determining the magnitudes of the principal hydraulic conductiviies, one must also determine their orientations. One way to approach this problem is with the concept of directional hydraulic conductivity. There are at least two ways of defining directional hydraulic conductivity: hydraulic conductivity in the direction of flow and hydraulic conductivity in the direction of hydraulic gradient (Marcus, 1962, and Bear, 1972). Hydraulic conductivity in the direction of flow, which is more widely used, is the ratio of the specific discharge to the component of hydraulic gradient in the direction of the flow. Hydraulis conductivity in the direction of hydraulic gradient was defined by Scheideger (1960) as the ratio of the component of the specific discharge in the direction of hydraulic gradient to the magnitude of the hydraulic gradient. The two definitions do not yield the same results.

Let $B_{1}, \beta_{2}, B_{3}$ denote the angles between the direction of specific discharge and the three principal axes $x, y$, and $z$, respectively. The relation between the hydraulic conductivity in the direction of flow $k_{d}$ and the values of principal hydraulic conductivity $K_{x}, K_{y}, K_{z}$ is:

$$
\frac{1}{k_{d}}=\frac{\cos ^{2} \beta_{1}}{k_{X}}+\frac{\cos ^{2} \beta_{2}}{k_{y}}+\frac{\cos ^{2} \beta_{3}}{k_{z}}
$$


Consider a vector $r$ along the direction of flow. Components of this vector along the coordinate axes are $x=r \cos \beta 1, y=r \cos \beta_{2}, z=r \cos \beta_{3}$. Substituting for $\cos \beta_{1}, \cos \beta_{2}$, and $\cos \beta_{3}, \mathrm{Eq} \cdot(2-52)$ yields

$$
\therefore \quad \frac{r^{2}}{k_{d}}=\frac{x^{2}}{k_{x}}+\frac{y^{2}}{x_{y}}+\frac{z^{2}}{x_{z}}
$$

If the length of vector $r$ is $r=\sqrt{k_{d}}$, the end point, traced from the origin, will coincide with an ellipsoid

$$
\frac{x^{2}}{x_{x}}+\frac{y^{2}}{x_{y}}+\frac{z^{2}}{x_{z}}=1
$$

The semi axes of this ellipsoid are $\sqrt{K_{x}}, \sqrt{K_{y}}$, and $\sqrt{K_{2}}$, in the $x, y$, and z directions, respectively. Hence, with the principal hydraulic conductivities and their orientation, one can define the ellipsoid of hydraulic conductivity. Once the ellipsoid is defined, hydraulic conductivity in any direction of flow can be easily obtained from $K_{d}=r^{2}$. Conversly, if hydraulic conductivity in the direction of flow can be determined for enough orientations to define the ellipsoid, the directions and magnitudes of the principal hydraulic conductivities can be exsily obtained. The direction and magnitudes of the principal hydraulic conductivities must be known for numerical modeling. The theory and procedure for obtaining these data are presented bilow. Hsieh et al. (1982) have also reported on a method of determining three dimensional anisotropic permeability of fractured rocks. The following derivation; however, has been developed independent of their work.

The drawdown at point $B(x, y, z)$ of Fig. 2.9 due to a point sini located at $A\left(x^{\prime}, y^{\prime}, z^{\prime}\right)$ discharging at a continuous rate $Q$ from an infinjte homogeneous anisotropic media may be written as 


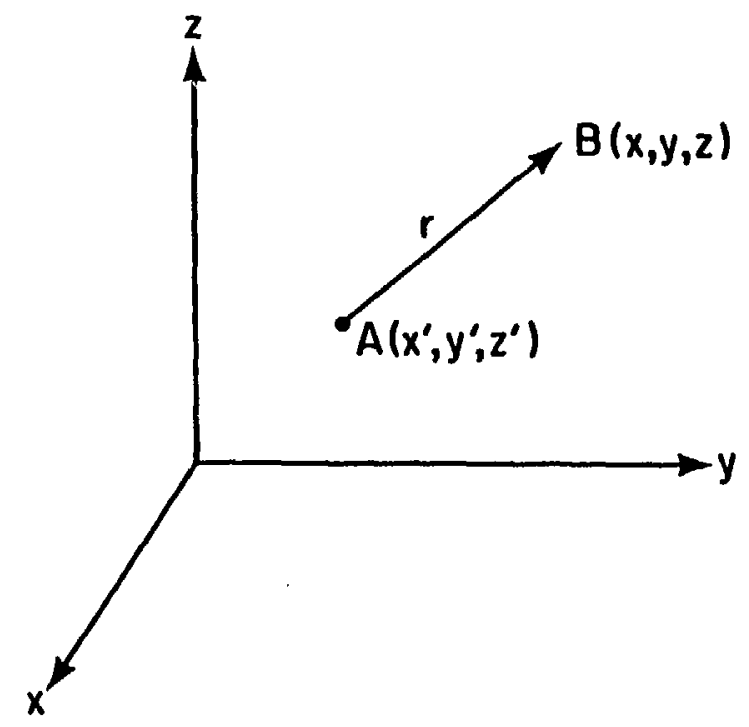

Fig. 2.9 Diagram of the position of the measurement point $B$ and a flow line emanating from the source $A$. 
$s=\frac{u}{4^{\pi}\left(k_{x} k_{y} k_{z}\right)^{1 / 2}}$

$$
\frac{\operatorname{erfc} \sqrt{\frac{5}{4 t}\left[\frac{\left(x-x^{\prime}\right)^{2}}{x_{x}}+\frac{\left(y^{\prime} y^{\prime}\right)^{2}}{x_{y}}+\frac{\left(z \cdot z^{\prime}\right)^{2}}{x_{z}}\right]}}{\left(\frac{\left(x-x^{\prime}\right)^{2}}{x_{x}}+\frac{\left(y-y^{\prime}\right)^{2}}{x_{y}}+\frac{\left(z-z^{\prime}\right)^{2}}{x_{z}}\right)^{1 / 2}}
$$

where

$$
\begin{aligned}
K_{x}, K_{y}, K_{z}= & \text { principal hydraulic conductivities in the direction of the } \\
& \quad \mathbf{x}, y, z \text { axes } \\
t & =\text { time elapsed since the start of production } \\
s_{5}= & \text { specific storage of the medium } \\
\text { erfc }(x)= & \text { complementary error function }
\end{aligned}
$$

If $A B$ represents a section of a flowline eranating from point $A$ and passing through point $B$, the following expression relates $K_{x}, K_{Y}$, and $k_{z}$ to the directional hydraulic conductivity $\mathbf{k}_{\mathrm{d}}$

$$
\frac{r^{2}}{K_{d}}=\frac{\left(x-x^{\prime}\right)^{2}}{K_{x}}+\frac{\left(y-y^{\prime}\right)^{2}}{K_{y}}+\frac{\left(z-z^{\prime}\right)^{2}}{K_{z}}
$$

where

$\mathbf{r}=$ the distance between points $\mathrm{A}$ and $\mathrm{B}$

$\mathrm{K}_{\mathrm{d}}=$ directional hydraulis conductivity in the direction of flow along $A B$

Substituting from Eq. (2-56) into Eq. (2-55) gives

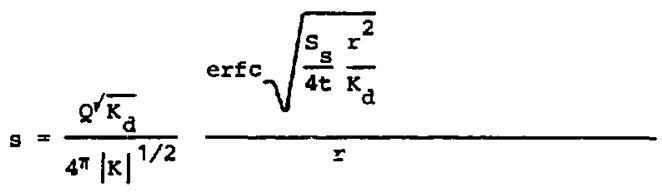

where 
$\mathrm{K}=\left(\mathrm{Kx} \mathrm{KY}_{\mathrm{Y}} \mathrm{K}\right.$ ) is the determinant of the hydraulic conductivity matrix and is an invarient.

Introducing the following dimensionless parameters:

$$
\begin{aligned}
& t_{D}=\frac{t k_{d}}{s_{s} r^{2}} \\
& s_{D}=\frac{4 \pi r s / \sqrt{k / / k} d}{Q}
\end{aligned}
$$

Equation $(2.5 /)$ becomes

$$
s_{D}=\operatorname{erfc} \sqrt{1 /\left(4 t_{D}\right)}
$$

Figure 2.10 is a type curve thowing the variation of $s_{D}$ versus $t_{D}$ as given by equation $(2-60)$.

To find $\mathrm{K}_{\mathrm{d}}$ in a given direction, one measures the change in hydraulic head at one point in the medium, due to a continuous point source at another point in the medilm. Figure 2.11 shows the arrangement: of such a test. A short interval, about 2 to 3 feet long, is packed off in well $A$ and water is injected or produced at a constant rate, $Q$, through the packed-off section. The center point, $c$, of this packed-off section is the approximate location of the point source or sink. The changes of hydraulic head, $s$ with time is measured in another short isolated interval in well B. D is the center point of the isolated interval in well $\mathrm{B}$. To calculate he hydraulic conductivity $K_{\mathrm{d}}$ in the direction of line $C D$ :

- Make $\log \log$ plots of variations of $s$ with $t$, measured at the point D, with the same scale as the type curve in Fig. 2,10 .

- Use superposition to find the best match with the type curve and then find the dual coordinates of a match point. 


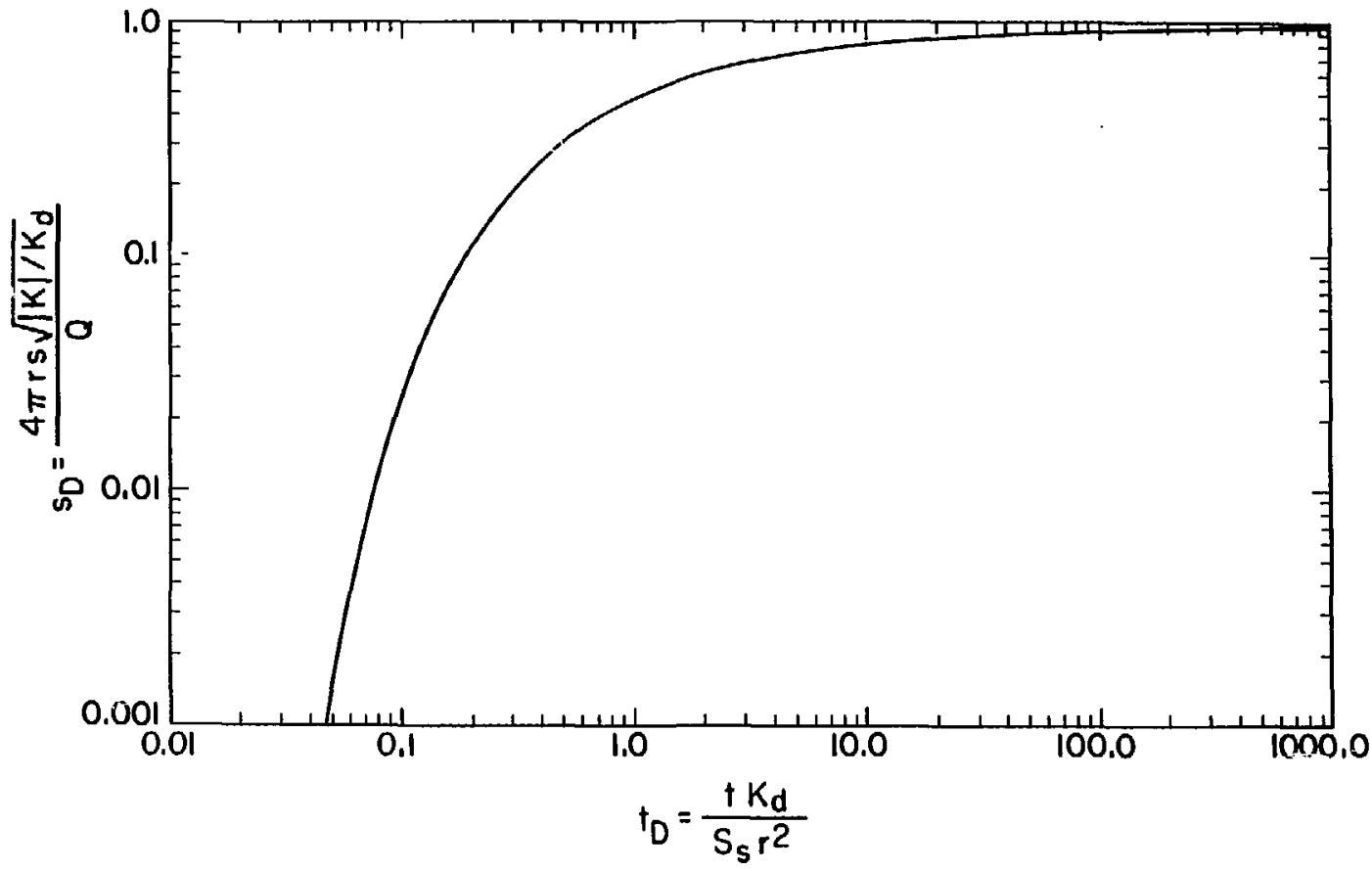

$\stackrel{1}{t}$

XBLE35-1829

Fig. 2.10 The type curve showing variation of $s_{D}$ versus $t_{D}$ as given by Equation (2-35). 


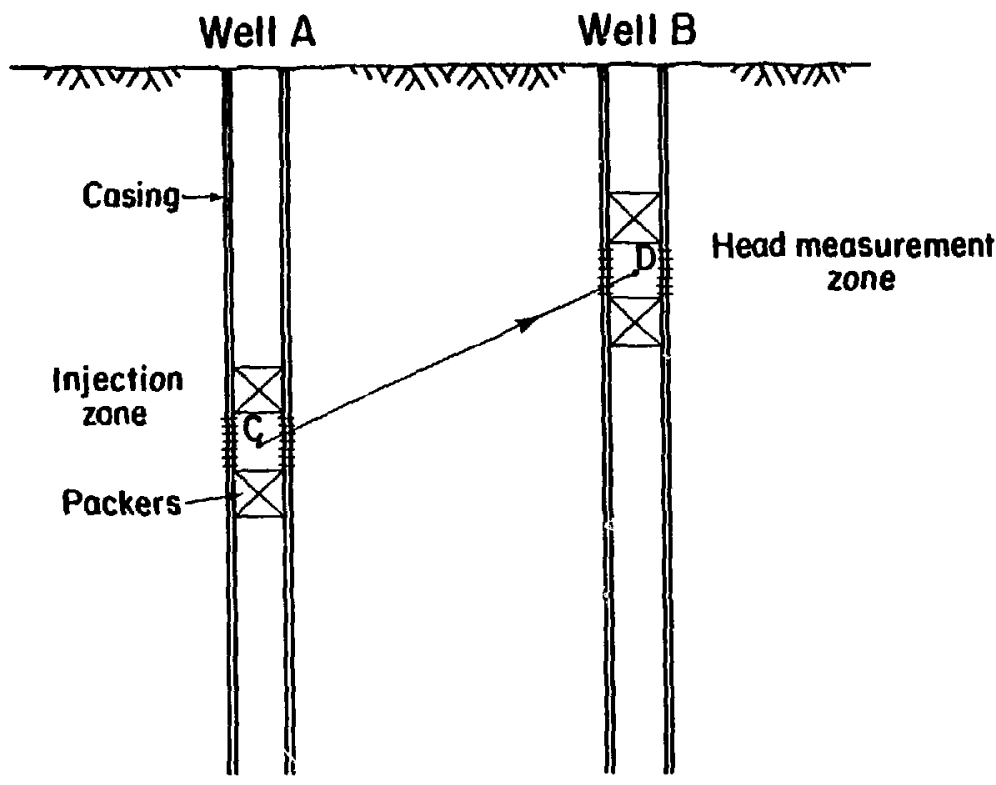

XBL 835-1830

Fig. 2.11 Diagram of an arrangement of a test based on the concept of directional hydraulic conductivity. 
- Knowing the values of $r, q$, and the coordinxices of the riton point $t, s, t_{D}, s_{D}$, find the ratio $k d / k \mid$ from.

$$
\frac{K_{d}}{|K|}=\left(\frac{4 \pi r s}{Q s_{D}}\right)^{2}
$$

- Calculate the ratio of $\mathrm{K}_{\mathrm{d}} / \mathrm{s}_{\mathrm{g}}$ from

$$
\frac{k_{d}}{s_{s}}=\frac{r^{2} t_{D}}{t}
$$

Although $|K|$ is a constant for a given homogeneous medium, it's magnitude is unknown. Further analysis is required to find the value of $|\mathrm{K}|$ as well as the values of all components of the hydraulic conductivity matrix.

If one selects a coordinate system $x, y, z$ which does not necessarily coincide with the principal directions of the hydraulic conductivity, then Eqs. $(2-52)$ and $(2-53)$ may be generalized:

$$
\begin{aligned}
& \frac{\cos ^{2} \beta_{1}}{k_{x x}}+\frac{\cos ^{2} \beta_{2}}{k_{Y Y}}+\frac{\cos ^{2} \beta_{3}}{k_{z z}}+\frac{2 \cos \beta_{1} \cos \beta_{2}}{k_{x y}}+\frac{2 \cos \beta_{1} \cos \beta_{3}}{k_{x z}} \\
& +\frac{2 \cos \beta_{2} \cos \beta_{3}}{k_{y z}}=\frac{1}{k_{d}} \\
& \frac{x^{2}}{k_{X X X}}+\frac{y^{2}}{k_{y Y}}+\frac{z^{2}}{k_{z z}}+\frac{2 x y}{k_{x y}}+\frac{2 y z}{k_{X z}}+\frac{2 y z}{k_{y z}}=\frac{r^{2}}{k_{d}}
\end{aligned}
$$

Here $B_{1}, B_{2}$, and $B_{3}$, represent the angles between the direction of specific discharge and the axes $x, y$, and $z$, respectively. If the constant $(1 /|k|)$ is denoted by "a", dividing both sides of equation(2-64) by "a" would give

$$
\frac{x^{2}}{a K_{x x}}+\frac{y^{2}}{a K_{y Y}}+\frac{z^{2}}{a K_{z z}}+\frac{2 x y}{a K_{X Y}}+\frac{2 x z}{a K_{x z}}+\frac{2 y z}{a K_{y z}}=\frac{r^{2}}{a K_{d}}
$$


Again, if the length of vector $r$ is $r=\sqrt{a K_{d}}$, the end point of such vector traced from the origin will coincide with an ellipsoid whose equation is

$$
\frac{x^{2}}{a K_{x x}}+\frac{y^{2}}{a K_{y y}}+\frac{z^{2}}{a K_{z z}}+\frac{2 x y}{a K_{x y}}+\frac{2 x z}{a K_{x z}}+\frac{2 y z}{a K_{y z}}=1
$$

To construct such an ellipsoid one needs to find six unknowns: akxx' $a k_{y y}, a k_{z z}, a k_{x y}, a K_{x z}$, and $a k_{y z}$. To calculate these it is necessary to establish and solve six linearly independent equations in terms of the unknowns. Therefore, one must design a test to determine the values of $\left(K_{d} /|k|\right)=a K_{d}$ in six appropriately selected lirections. Now one can choose an arbitrary coordinate system with it's origin located at the source or the sink. The coordinates of each of six head measurement points idesignated by $x_{i}, y_{i}, z_{i}$. The distances of those points from the source or the sink are given by $r_{i}$, and the calculated directional hydraulic conductivities by $\left(K_{d}\right)_{i}$. Equation $(2-65)$ can now be written:

$$
\frac{x_{i}^{2}}{a K_{x x}}+\frac{y_{i}^{2}}{a K_{y y}}+\frac{z_{i}^{2}}{a K_{z z}}+\frac{2 x_{i}^{y} y_{i}}{a K_{X Y}}+\frac{2 x_{1} z_{i}}{a K_{x z}}+\frac{2 y_{i} z_{i}}{a K_{y z}}=\frac{r_{i}^{2}}{a\left(K_{d}\right)}
$$

The solution to the above six equations give $a k_{x x}, a K_{y y}, a k_{z z}$, $a k_{x y}$, $a_{x z}, a k_{y z}$. Recalling that the hydraulic conductivity matrix is symmatric, the entire matrix of $\mathrm{ak}_{\mathrm{ij}}$ can be developed. Since

$$
\operatorname{det}\left[a K_{i j}\right]=a^{3}|k|=a^{2}
$$

the value of $a=(1 /|k|)$ may be obtained from

$$
a=\left(\operatorname{det}\left(a K_{i j}\right)^{1 / 2}\right.
$$


once the value of a is found, the hydraulic conductivity matrix for the teat zone is easily obtained from value of $a k_{1 j}$.

\subsubsection{Single Well Teats}

Estimating vertical permeabllity of the producting formations by single well tests has been customary in the petroleum industrles for a long time (Burns, 1969; Prats, 1970; Hirasaki, 1974). The theory behind these tests is based on the pressure response of a homogeneous, anisotropic porous medium due to a point or line source. The major assumptions usually used in the development of these tests are that the vertical hydraulic conductivity is one of the principal conductivities, and that conductivity in the bedding plane is independent of direction. With these assumptions in mind, if conductivity is measured in the vertical direction, the value of $\sqrt{|\mathrm{K}| / \mathrm{K}_{\mathrm{d}}}$ in equation (2-59) reduces to $k_{r}$. This will simplify the formulation and by arranging both injection and monitoring zones within a single well, one can easily estimate the value of vertical conductivity $\mathrm{k}_{\mathrm{z}}$. For more detailed discussion abour single well tests the reader is referred to Burns (1965), Prats (1970), Hirasaki (1974), and Javandel (1983). 
1. Tests based on the theory of partial penetration:

Weeks' Method

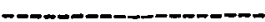

1. Homogeneous, confined and laterally extensive aquifer.

2. Hydraulic conouctivity perpendicular to bedding plane is a principal hydraul conductivity.

3. Horizontal hydraulic conductivity in the bedding plane is independent of direction.

direction

All the Week's Assumptions except for the following holds:

Hydraulis conductivity in the bedding plane could be a function of direction.
Determines both radial and vertical conductivity of an aquifer which is either porous medium or can be substituted by an equivalent porous medium. Determines the full Hy draulic conductivity tensor of the aquifer.
Both methods are useful

\section{for formations with}

relatively high hy-

draulic conductivity.

K should be of the

order of $10^{-7} \mathrm{~m} / \mathrm{s}$ or

larger. 
2. Tests based on the theory of leaky aquifers

Hantush - Jacob solution

Hantush modified solution

witherspoon and Neuman Ratio Method
Both aquifer and aquitard are homogeneous andisotropic.

Flow is essentially horizontal in the aquifer and vertical in the aquitard.

No drawdown occurs at the top of the aquitard due to punping in the aquifer.

Leakage into the pumped aquifer is proportional to potential drop across the aquitard. In other words the storage capacity of the aquitard is negligible.

Except for considering storage capacity to the aquitard the rest of assumptions are the same as Hantush-Jacob solution.

Vertical flow in the aquiclude and horizontal flow in the aquifer.

Draw down in the aquifer is approximated by the Theis solution.

The effect of pumping the aquifer does not reach the top of the aquiclude.
Determines hydraulic conductivity of the aquifer and the aquitard when the aquitard is relatively thin, rigid and relatively permeable.

Determines the product of conductivity and storage coefficient for the aquitard.

Determines the ratio of conductivity and specific storage of the aquiclude.
This method does not differentiate the offect of water seeping into aquifer from the aquitard above or below.
If the aquifer in enbedded between two aquitarda with different hydraulic properties thi method can not differentiate the hydraulic properties of individual aquitards. 
3. Test based on the concept of airectional hydraulic conductivity

Multiple well Test

Single Well Tests
The testing medium is approximated as a homogeneous anisotropic porous medium.

Injection inteval is 60 small that can be approxinated as a point source.

Hydraulic conductivity in the direction perpendicular to the bedding plane is one of the principal values of conductivity. and in the bedding plane conductivity is independent of dirtction.
Determines the magnitude and directions of principal hydraulic conductivities of a

formation which can be represented by a homogeneous anisotropic porous medium.

Determines vertical hy draulic conductivity of a relatively low pe:meable material which can be approximated as a homogeneous anisotropic porous medium.
The testing period is reasonable only for relatively permeable materials. For materials with very low permeability the length of test may be so long that the test is no longer practical.

The value of hydraulic conductivity calculated by this method corresponds to a small volume of rock located in the vicinity of the testing zone. 


\section{COMPARISON OF AVAILABLE TEChNIQUES}

In this section we compare the several methods of determining hydraulic conductivity so that the method, or methods, appropriate to the BwIP site can be selected. -

Methods based on directtonal hydraulic conductivity are the only ones which do not assume a knowledge of the orientation of the principal hydraulic conductivities. The methods based on the theory of partial penetration and the theory of leaky aquifers all assume that one of the principal hydraulic conductivity directions is perpendicular to the bedding plane. When the beddirg plane is hortzontai, this principal direction will be vertical. The other two principal hydraulic conductivity directions must, therefore, be in the bedding plane. In the Shao-McKee method, the principal hydraulic conductivity directions are determined as part of the analysis. In Heeks' method, hydraulic conductivity on the bedding plane is assumed to be the same in all directions.

AII of the methods based on the theory of leaky aquifers consider the aquitard or aquiclude to be homogrneous and isotropic. Vertical and horizontal conductivities are, therefore, assumed to be equal and independent of direction. As a result, if the fracture configuration within the aquitard causes the medium to be anisotropic with unkown orientations of principal hydraulic conductivity, leaky aquifer methods yield indeterminate results. Only the tests cised on the concept of directional hydraulic conductivity would provide all of the information required for numerical modeling.

Another major difference between these methods is that those based on the theory of partial penetration and those based on the concept of direc- 
tlonal hydraulic conductivity are performed directly in the horizon whose hydrologic properties are being determined. By comparison, those tests based on the theory of leaky aquifers are performed indirectly by pumping in an adjacent aquifer. This difference infuences the volume of rock over which the hydrologic properties can be evaluated.

When tests based on cencepts of directional hydraulic conductivity are performed directly in the low permeability rock, the zone of influence of the test is very small. This is true even in deep formations where it is possible to generate large differential heads between the pumping well and the surrounding rock; large head differentials produce large changes in the effective stress in the rock around the borehole. If the head difference is produced by pumping, tire changes in stress may close fractures. Conversely, if water is injected under large differential heads, the fractures may open. These changes in fracture aperature can produce significant changes in the hydraulic conductivity of the rock mass and distort the test results. Such effects limit the head difference that can be used in the test and will generally limit the zone of influence of the test to a smaIl volume of rock around the test hole.

In the case of tests based on the theory of leaky aquifer, water is pumped from, or tnjected into, a permeable horizon to cause a pressure drop or increase, over a relatively large area. The effects of such disturbances are then monitored within aquitards, or aquicludes, surrounding the pumped horizon. The volume of rock within the ciquitard or aquiclude that is influenced by this type of test is much larger than in the other methods. Therefore, values of hydraulic conductivity obtained by leaky aquifer methods will 
generally be more representative of the large scale average properties of the aquitards or aquicludes.

Of the leaky aquifer tests, the Hantush-Jacob $(r / B)$ and the modified Hantush method are the simplest to design and operate. These tests require measurement of only drawdowns in the pumping horizon. Instrumentation is standard and does not require special development. In the ratio method, however, measurement of drawdowns within the aquitard or aquiclude is also required. Instrumentation for this test is more complex and may require specially developed equipment. However, it has the advantage that the hydraulic properties of the low permeability strata both below and above the pumped horizon can be evaluated. This cannot be done with the other leaky aquifer methods. The capbility to discriminate between the properties of the overlying and underlying horizons is particularly important if thier hydrological properties or thicknesses are significantly different. 


\section{RECOMMENDED METHODS}

The cholce of the testing method for estimating the hydraulic conductivity tensor, in general, and the vertical hydraulic conductivity, in particular, of a geological horizon is a function of several factors. These factors include the magnitude of the hydraulic conductivity of the medium and available knowlege of the orientations of the principal hydraulic conductivity. Suitability and availability of a sufficient number of wells and their positions with respect to each other is another factor which is of planning and economic concern. If one is interested in testing a horizon with a relatively low hydraulic conductivity, the availability of an aguifer either above or below that horizon is also an important element in the choice of testing method.

Table 4-1 gives preliminary measurments of the hydraulic conductivites of basalt horizons from four boreholes on the BWIP sice. This table shows that the effective hydraulic conductivity of individual flow tops and flow bottoms varies over four to six orders of magnitude. For example, the Cohassett flow bottom at boreho: e RRL2 has a relatively high conductivity of $3.5 \times 10^{-5} \mathrm{~m} / \mathrm{s}$; the same horizon at RRL6 and DC-16A has conductivities that are 5 to 6 orders of magnitude lower. It is difficult, therefore, to suggest a specific test that will, under all circumstances, be suitable for a given horizon when such large lateral heterogeneit; is observed in its hydraulic properties.

Where a well developed collumnar structure is present in a basalt flow, Se orientation of the vertical principal hydraultc conductivities could be brtipipated prior to testing. In other parts of a basalt flow, fracturing 
Table 4-1. Hydraulic conductivity and effectlve thickness of some Basalt flow tops and bottons at the BWIP site.

\section{Cohassett Flow Bottom}

\section{Borehole. Thickness}

m $\mathbf{f}$

23.578

$20 \quad 67$

RRI -6

RRL- 14

$D C-16 A$

$R R I-2$

$23.5 \quad 77$

19.264

\section{effective $K$}

$$
\mathrm{m} / \mathrm{s}
$$

$$
\text { ft/day }
$$

$$
9.87
$$

$3.5 \times 10^{-5}$

$$
1.5 \times 10^{-5}-1.5 \times 10^{-4}
$$

$1.3 \times 10^{-3}-1.3 \times 10^{-2}$

$10^{-9}-10^{-8}$

$1.5 \times 10^{-5}-1.5 \times 10^{-4}$

\section{Gohassett Flow Top}

\section{Borehole}

Thickness

$$
\text { m } \quad \mathbf{f}
$$

4.214

RRL-2

RRL-6

$R R L-14$

$D C-16 A$

Borehole

Thiokness

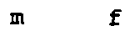

E

m/s

$$
\text { RRL-2 }
$$

47

157

$28.5 \quad 95$

$21.6 \quad 72$

RRL-14

DC- $16 \mathrm{~A}$

$\begin{array}{llr}\text { RRL-6 } & 28.5 & 95 \\ \text { RRL-14 } & 21.6 & 72 \\ \text { DC-16A } & --- & --\end{array}$

$\mathrm{m} / \mathrm{s}$

$2.5 \times 10^{-9}$

$10^{-12}-10^{-11}$

$10^{-7}-10^{-6}$

$2 \times 10^{-7}$

3. Untanum Flow Top

\section{effective $K$}

$f t / d a y$

$7 \times 10^{-4}$

$10^{-7}-10^{-6}$

$2.8 \times 10^{-?}-2.8 \times 10^{-1}$

$5.8 \times 10^{-2}$

\section{Effective $K$}

$$
\text { ft/day }
$$

$0.5-3.1$

$10^{-3}-10^{-2}$

$10=2-10^{-1}$

$10-B-10^{-7}$ 
may not be essentially orthogonal and will generally not be oriented in vertical or torizontal directions. Also, different fracture sets may have different properties. Thus, the principal directions of hydraulic conductivity in flood basalts may not always be established before testing. As a result, the selection of test method must take into account the geologic structure and range of permeabllity anticipated for the test horizon. In some cases, further research will be needed to develop appropriate testing technigues.

Below we consider the applicability of test methods to measurement of the hydraulic conductivity of twc rock mass types found in the basalts at the BWIP site.

\section{1 Dense Flow Interiors}

Typical values of hydraulic conductivity measured in the entablatures and colonnades of the Cohassett and Umtanum flows are given in Table 4-2; they are equal or liess than $10^{-12} \mathrm{~m} / \mathrm{s}$. For this range of hydraulic conductivity, the only method among the previously discussed techniques, which might be applied directly within the horizon is the one based on the concept of directional hydraulic conductivity. We examined the feasibility of applying this method to the Cohassett entablature at the DC-16 cluster boreholes.

Becauge the vertical conductivity of the Cohassett entablature is not known at DC-16, the entablature is assumed to be isotropic. The minimum distance between any two of the available boreholes in this cluster is the distance between $\mathrm{DC}-16 \mathrm{~A}$ and $\mathrm{B}$, which is about $15 \mathrm{~m}$. If the specific storage is $10^{-5} \mathrm{~m}^{-1}$, the ratio of the drawdown to the injection rate $(\mathrm{s} / \mathrm{Q})$ after 30 and 180 days of testing may be obtained from equation(2-57) 
Table 4-2. Hydraulic conductivities estimated from testa performed is. the dense Interiors of the Cohassett and rtanum flows.

\section{Cchasgett. Entablature}

Borehole

RRL-2

RRI-6

60

Thickness

ft

II

20.7

69

203

\section{Effective K}

m/s

ft/day

$2.3 \times 10^{-13}$

$6 \times 10^{-8}$

$10^{-16}-10^{-13}$

$10^{-10}-10^{-7}$

\section{Untanum Entablature/Colonnade}

Borehole

RRL-6

RRL - 2
Thickness

m ft

33

13

ft

111

43

\section{Effectlve $K$}

m/s

ft/day

$10^{-16}-10^{-15}$

$10^{-10}-10^{-9}$

$10^{-12}$

$10^{-6}$ 


$$
\begin{aligned}
\frac{S}{Q} & =\frac{\operatorname{erfc} \sqrt{\frac{s}{4 t} \frac{r^{2}}{K}}}{4 \pi r} \\
& =\frac{\operatorname{erfc} \sqrt{\frac{10^{-5} \times 225}{4 \times 2.6 \times 10^{6} \times 10^{-12}}}}{4 \pi \times 10^{-12} \times 15}=0.27 \times 10^{-85} \mathrm{~m} / \mathrm{m}^{3} / \mathrm{s}
\end{aligned}
$$

for $t=30$ days and

$$
=\frac{\operatorname{erfc} \sqrt{\frac{10^{-5} \times 225}{4 \times 15.6 \times 10^{6} \times 10^{-12}}}}{4_{\pi} \times 10^{-12} \times 15}=0.16 \times 10^{-6} \mathrm{~m} / \mathrm{m}^{3} / \mathrm{s}
$$

for $t=180$ days.

For practical values of $\mathcal{Q}$, the magnitude of drawdown is so small that its measurement is impossible.

One can conclude that if the entablature has a hydraulic conductivity on the order of $10^{-12} \mathrm{~m} / \mathrm{s}$ or less, and if $s_{s}=10^{-5} \mathrm{~m}^{-1}$, no direct measurement of the entire hydraulic conductivity tensor can be made using the present spacing of the wells in the DC-16 cluster.

Table 4-3 presents the ratio of $s / Q$ for different values of $r, t$, and K calculated from Eq. (2-57). The table shows that the directional conductivity test could be applied in a low permeability entablature if the distance between the injection zone and the monitoring points around it is on the order uf 2 to $4 \mathrm{~m}$. One should note, however, that the data obtalned from such a test could be representative of a relatively small volume of the rock. 
Table 4-3. Values of $s / Q$ for several values of $r$, $t$ and $x$ calculated From equation (2-57).

\begin{tabular}{llll}
$r, \mathrm{~m}$ & $t$, days & $\mathrm{k}, \mathrm{m} / \mathrm{s}$ & $8 / \mathrm{g} / \mathrm{m}^{3} / \mathrm{a}$ \\
15 & 30 & $10^{-12}$ & $.27 \times 10^{-85}$ \\
15 & 180 & $10^{-12}$ & $.16 \times 10^{-6}$ \\
4 & 30 & $10^{-12}$ & $.41 \times 10^{3}$ \\
4 & 160 & $10^{-12}$ & $.45 \times 10^{7}$ \\
\hline
\end{tabular}


An alternative approach which could be applied to the DC-16 cluster is to use two separate single well tests within the entablature to measure the vertical component of the conductivity (Javandel, 1983). The horizontal values of hydraulie conductivity in such a horlzon may be obtained by the modified slug test method presented by Bredehoeft and Papadopulos (1980) subject to corrections suggested by Neuzil (1982). However, if the conductivities are vary low, the results of these tests will represent a relatively small volume of rock. Also, these methods cannot provide the complete Information on directional conductivities that would be available from a directional hydraulic conductivity test.

An alternative to direct testing within the entablature is to use indirect techniques, such as the ratio method, that are based on the theory of leaky aquifers. The use of the ratio method, however, requires the assumption that the hydraulic conductivity within the entablature is isotropic. In this test, one of the permeable horizons above or below the entablature is pumped and the drawdowns both in the pumping horizon and the adjacent entablature are monitored. The feasibility of applying the ratio method to find the hydraulic conductivity of the entablature can be calculated. From Table 4-1, the most permeable horlzon in the upper part of Grande Ronde formation in the DC-16 cluster area is the Cohassett flow top whose hydraulic conductivity $1 \mathrm{~s}$ of the order of $10^{-7} \mathrm{~m} / \mathrm{s}$. With this value of conductivity the ratio of drawdorm to the rate of discharge $1 \mathrm{n}$ such an aquifer 15 m from the pumping well (the distance between $D C-16 A$ and $B$ ), after one month of pumping, may be obtained as follows:

$$
\begin{aligned}
& r=15 \mathrm{~m} \\
& S_{g}=10^{-5} \mathrm{~m}^{-1}
\end{aligned}
$$




$$
\begin{aligned}
t & =2.6 \times 10^{6} \mathrm{sec} \\
x & =10^{-7} \mathrm{~m} / \mathrm{s} \\
b & =10 \mathrm{~m}, \text { aquifer thickness } \\
u & =\frac{r^{2} s_{s}}{4 t \mathrm{~K}}=.21 \times 10^{-2} \\
W(u) & =5.64 \\
\frac{\Delta h}{Q} & =\frac{W(u)}{4 \mathrm{~Kb}}=0.44 \times 10^{6} \mathrm{~m} / \mathrm{m}^{3} / \mathrm{s}
\end{aligned}
$$

If $D C-16 B$ were pumped at a production rate of $3 \times 10^{-4} \mathrm{~m}^{3} / \mathrm{s}$, drawdown would be about $130 \mathrm{~m}$ in the aquifer at DC-16A after one month. The drawdown within the entablature in DC-16A may be obtained as follows:

$$
\begin{aligned}
K^{\prime} & =10^{-12} \mathrm{~m} / \mathrm{s} \\
t & =2.6 \times 10^{6} \mathrm{sec} \\
s_{s} & =10^{-5} \mathrm{~m}^{-1} \\
z & =5 \mathrm{~m}
\end{aligned}
$$

From Fig. 2.6, $s^{\prime} \mathrm{s}^{\prime}<10^{-5}$ or $\mathrm{s}^{\prime}<0.001$.

The above calculation shows that the drawdown within the entablature, 5 m from the pumped horizon, is less than $1 \mathrm{~mm}$, which is difficult to measure. However, if the pumping time is extended to 3 to 4 months, or if the specific storage $s_{s}$ is $1 / 5$ of what was assumed above, then the magnitude of drawdown within the entablature would fall within a range that can be easily measured. If tive magnitude of $s_{s}$ could be reliably established, and if there were no changes in hydraulic head in the entablature, the test would at least establish an upper limit for conductivity. Infortunately, $s_{s}$ ' is not usually known. 
Figure 4.1 presents the ratio of drawdown in the entablature to drawdown in the pumped horizon as a function of the vertical hydraulic conductivity of the entablature. The figure shows results for two different values of specific storage, $10^{-6}$ and $10^{-7} \mathrm{~m}^{-1}$, and two test periods of 14 and 20 days. It is apparent that if the specific atorage of the entablature is less than or equal to $10^{-6} \mathrm{~m}^{-1}$, then the magnitude of the drawdown in that horizon, 15 of from the pumping well, after 3 weeks of steady pumping at the rate of $3 \times 10^{-4} \mathrm{~m}^{3} / \mathrm{s}$ would be more than $10 \mathrm{~cm}$. This is an amount that can be easily measured.

Because it is difficult to predetermine the best test method for the conditions in the formation, the best approach would be to install the pump test equipment so that the data obtained could be analyzed by several techniques. For example, if we obtain a good set of data from the ratio test, these data can also be avalysed by Hantush's $\underline{b}$ and $I / B$ methods.

\subsection{Flow Tops and Bottoms}

Because the flow tops and bottoms are expected to be more permeable than the adjacent dense interiors of the basalt flows, only direct testing techniques can be used within these horizons. If the hydraulic conductivity of a flow top is on the order of $10^{-7} \mathrm{~m} / \mathrm{s}$ or more, then two alternatives are possible. (1) If one has reason to believe that two of the principal directions of hydraulic conductivity lie on the bedding plane and the third is perpendicular to that plane, the Shao-Mckee method is recommended. (2) If one does not know how the principal hydraulic conductivities are oriented, then the test based on the concept of directional hydraulic conductivity is recommended. If the hydraulic conductivity is less than $10^{-7} \mathrm{~m} / \mathrm{s}$, the 


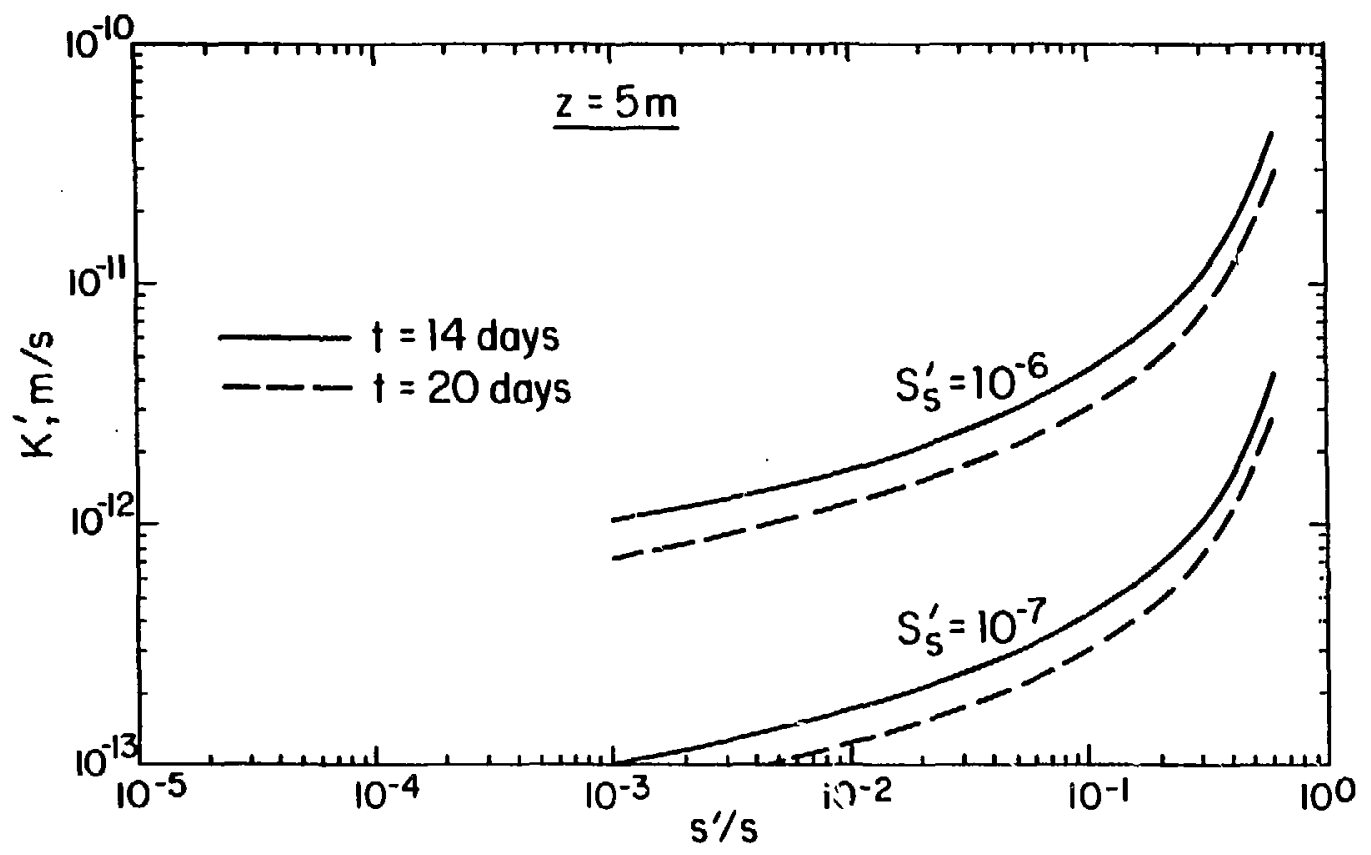

Fig. 4.1 The variation of ratio of drawrown in the entablature to drawdown in the pumped horizon $s^{\prime} / s$, versus $K^{\prime}$, the vertical hydraulic conductivity of the entablature. 
methods recommend in Section 4.1 for test performed directly within the entablature could be used. In general, conductivities within the flow tops are expectej to be sufficiently high that significant problem in field measurement will not be encountered. 
ACKNOWLEDGMENTS

The author would like to thank Drs. Charles Wilson and Dai Watkins of Lawrence Berikeley Laboratory for their careful review of this manuscript. The comments of Drs. Leo Leonhart and Frank Spane of Rockwell Hanford Operations are also acknowledged. 


\section{REFERENCES}

Bear, J., 1972. Dynamics of Fluids in Porous Media, American Elsevier, New York, 764 pp.

Bredehoeft, J.D. and S.S. Papadopulos, 1980. A method for determining the hydraulic properties of tight formations, water Resources Research. 16 (1), pp. 233-238.

Burns, W.A., Jr., 1969. New single well test for determining vertical permeability. Trans. AIME, vol. 246, pp. 743-752.

Glover, R.E., W.T. Moody, and W.N. Tapp, 1960. Till permeabilities as estimated from the pump-test data obtained during the irrigation well investigations, in Studies of Groundwater Movement, Bureau of Reclanation Tech. Memo 656.

Hantush, M.S., and C.E. Jacob, 1955. Non-steady radial flow in an infinite leaky aquifer, Trans. Amer. Geophys. Union, 36, pp. 95-100.

Hantush, M.S., 1956. Analysis, of data from pumping tests in leaky aquifers, Trans. Amer. Geophy. Union, 37, pp. 702-714.

Hantush, M.S., 1957. Nonsteady flow to a well partially penetrating an infinite leaky aquifer, Proceeding, Iragi Scientific Soc. vol. 1 . 10.

Hantush, M.S., 1960. Modification of the theory of leaky aquifers, Jour. Geophy. Res., 65(11), pp. 3713-3726.

Hantush, M.S., 1960 b. Tables of the Function H(u, S ), Document 6427, U.S. Library of Congress, Washington, D.C.

Hantush, M.S., 1961. Drawdown around a partiallY penetrating well, ASCE, $\underline{J}$. of Hydraulic Div. HY4, pp. 83-98.

Hantush, M.S., 1964. Hydraulics of Wells, in Advances in Hydroscience, Ven Te Chow, Ed. Academic Press, New York, Vol. 1.

Hirasaki, G.J., 1974. Pulse tests and other early transient pressure analyses for in-situ estimat on of vertical permeability, Trans. AIME, vo1. 257, pp. 75-90.

Hsieh, P.A., S.P. Neuman, and E.S. Simpson, 1982. A field method for determining three-dimensional anisotropic permeability of fractured rocks, Abstrat, Tran. American Geophysical union. 63(45), pp. 934.

Hubbert, M.K., 1940. The theory of groundwater motion, J. Geol., 48, pp. 785-944. A.1so in Theory of Groundwater Motion and Related Papers, Hafner, New York, 1969.

Jacob, C.E., 1946. Radial flow in a leaky artesian aquifer, Trans. Amer. Geophys. Union, 27, pp. 198-205. 
Javandel, I., 1983. Field Deternination of the Hydrologic Properties and Parameters that Control the Vertical Component of Groundwater soverent, Earth Sciences Divsion, Lawrence Berkeley Laboratory Report LBL-15050, in preparation.

Leahy, P.P., 1976. Hydraulic Characteristics of the Piney Point Aquifer and Overlying Bed Near Dover, Delaware: Delaware Geol. Survey Report of Invest. No. 26, p. 24 .

Lohman, S.W., 1972. Grounawater Hydraulics, USGs Professional paper 70B, 70 Pp.

Marcus, H., 1962. The permeability of a sample of an anisotropic porous media, J. Geophy. Res., 67(13), pp. 5215-5225.

Narasimhan, T.N.., 1968. Ratio method for determining characteristics of ideal, leaky and bounded aquifers., Bull. of Inter. Ass. of Sci. Hy= drology (13), pp. 70-83.

Neuman, S.P., 1966. Transient Behavior of an Aquifer with a Slightly Leaky Caprock, M.S. Thesis, University of California, Berkeley.

Neuman, S.P. and P.A. Witherspoon, 1968. Theory of flow in aquicludes adjacent to slightly leaky aquifers, Water Resources Jesearch, 4(1), pp. 103-112.

Neuman, S.P., and P.A. Witherspoon, 1969. Applicability of current theories of flow in leaky aquifers, water Resources Research 5(4), pp. 817-829.

Neuman, S.P., and P.A. Witherspoon, 1969a. Theory of flow in a confined two-aquifer systems, Water Resources Research, 5 (4), pp. 803-816.

Neuman, S.P., and P.A. Witherspoon, 1972. Field determination of the hydraulic properties of leaky multiple aquifer systems, Water Resource Research B(5), pp. 1284-1298.

Neuzil, C.E., 1982, On conducting the modified 'slug' test in tight formations, Water Resources Research, 18(2) pp. 439-441.

Parr, A.D., J.G. Melville, and F.J. Molz, 1983. HP41C and TI59 programs for anisotropic confined aquifers, Ground Water, $21(2)$, pp. 212-220.

Prats, M., 1970. A method for determining the net vertical permeability near a well from in-situ measurements, Trans. AIME, vol. 249, pp. 637-643.

Rose, $\omega ., 1983$. A note on the role played by sediment bedding in causing permeability anisotropy, J. Pet. Tech. Feb. pp. 330-332.

Scheidegger, A.E., 1960. The Physics of Flow Through Porous Media, The Mackitian Company, New York, 313 pp. 
Shao-Chin Hay and C.R. KcKee, 1982. In-situ determination of three-dimensional aquifer permeabilities, Ground Water, 20 (5), pp. 594-603.

U.S. Bureau of Reclamation, 1977. Groundwater Manual, A water Resources Technical Publication, pp. 480 .

Walton, W.C., 1960. Leaky artesian aquifer condition in Illinois, Illinois State Water Survey Rep. Invest., pp. 39.

Weeks, F.P., 1969. Determining the ratio of horizontal to vertical permeability by aquifer-test analysis, water Resources Research, 5(1), pp. 196-214.

Weeks, E.P., 1977. Aquifer tests - The state-of-the-art in hydrology, Proceedings of Invitational We11-Testing Symposium, October 19-21, Berkeley, California, Lawrence Berkeley Laboratory, LBL-7027, Pp. 14-26.

Witherspoon, P.A., T.D. Miller, and R.W. Donovan, 1962. Evaluation of underground gas-storage conditions in aquifers through investigations of groundwater hydrology, Trans. AIME, vol. 225, pp. 555-561.

Witherspoon, P.A., I. Javandel, S.P. Neunan, and R.A. Freeze, 1967. Interpretation of Aquifer Gas Storage Conditions from Hater Pumping Tests, American Gas Assoc., Inc., New York, New York, 273 pp.

Witherspoon, P.A., and S.P. Neuman, 1967. Evaluating a slightly permeable caprock in aquifer gas storage. I. caprock of inifinite thickness, Jour. of Petroleum Tech. pp. 949-955.

Wolff, R.G., 1970. Relationship between horizontal strain near a well and reverse water level fluctuation; Water Resources Research, 6(6), PP. $1721-1728$. 
This report was done with support from the Department of Energy. Any conclusions or opinions expressed in this report represent solely those of the author(s) and not necessarily those of The Regents of the Universiny of California, the Lawrence Berkeley Laboratory or the Department of Energy.

Reference to a company or product name does not imply approval or recommendation of the product by the Universily of California or the U.S. Department of Energy to the exclusion of others that may be suitable. 